\title{
Ab initio study of the CO-N2 complex: a new highly accurate intermolecular potential energy surface and rovibrational spectrum
}

Cybulski, Hubert; Henriksen, Christian; Dawes, Richard; Wang, Xiao-Gang; Bora, Neha; Avila, Gustavo; Carrington, Tucker; Fernández, Berta

Published in:

Physical Chemistry Chemical Physics

Link to article, DOI:

$10.1039 / \mathrm{c} 8 \mathrm{cp} 01373 \mathrm{j}$

Publication date:

2018

Document Version

Peer reviewed version

Link back to DTU Orbit

Citation (APA):

Cybulski, H., Henriksen, C., Dawes, R., Wang, X-G., Bora, N., Avila, G., Carrington, T., \& Fernández, B. (2018). $\mathrm{Ab}$ initio study of the CO-N2 complex: a new highly accurate intermolecular potential energy surface and rovibrational spectrum. Physical Chemistry Chemical Physics. https://doi.org/10.1039/c8cp01373j

\section{General rights}

Copyright and moral rights for the publications made accessible in the public portal are retained by the authors and/or other copyright owners and it is a condition of accessing publications that users recognise and abide by the legal requirements associated with these rights.

- Users may download and print one copy of any publication from the public portal for the purpose of private study or research.

- You may not further distribute the material or use it for any profit-making activity or commercial gain

- You may freely distribute the URL identifying the publication in the public portal 
See discussions, stats, and author profiles for this publication at: https://www.researchgate.net/publication/324589069

\section{Ab initio study of the CO-N 2 complex: A new highly accurate intermolecular potential energy surface and rovibrational spectrum}

Article in Physical Chemistry Chemical Physics · April 2018

DOI: 10.1039/C8CP01373J

CITATIONS

5

8 authors, including:

Hubert Cybulski

Nicolaus Copernicus University

30 PUBLICATIONS 426 CITATIONS

SEE PROFILE

Dawes Richard

Missouri University of Science and Technology

137 PUBLICATIONS 1,841 CITATIONS

SEE PROFILE

Some of the authors of this publication are also working on these related projects:

Matrix Isolation View project

Project quantum dynamics bound states View project
104

Christian Henriksen

Technical University of Denmark

24 PUBLICATIONS 194 CITATIONS

SEE PROFILE

Xiao-Gang Wang

Queen's University

111 PUBLICATIONS 2,300 CITATIONS

SEE PROFILE 


\title{
$A b$ initio study of the $\mathrm{CO}-\mathrm{N}_{2}$ complex: A new highly accurate intermolecular potential energy surface and rovibrational spectrum
}

\author{
Hubert Cybulski* \\ Institute of Physics, Faculty of Physics, Astronomy and Informatics, \\ Nicolaus Copernicus University in Torun, \\ Grudziadzka 5, 87-100 Torun, Poland \\ Christian Henriksen ${ }^{\dagger}$ \\ Department of Applied Mathematics and Computer Science, \\ Technical University of Denmark, \\ 2800 Kgs. Lynbgy, Denmark \\ Richard Dawes $\ddagger$ \\ Missouri University of Science and Technology, Rolla, MO 65409, USA \\ Xiao-Gang Wang§ ${ }^{\S}$ Neha Bora, Gustavo Avila, and Tucker Carrington Jr. \\ Chemistry Department, Queen's University, \\ Kingston, Ontario KrL 3N6, Canada \\ Berta Fernández** \\ Department of Physical Chemistry, University of Santiago de Compostela \\ 15782 Santiago de Compostela, Spain
}

\footnotetext{
* Electronic mail: hubert@fizyka.umk.pl, hcybulski@gmail.com

$\dagger$ Electronic mail: chrh@dtu.dk

$\ddagger$ Electronic mail: dawesr@mst.edu

$\S$ Electronic mail: xgwang.dalian@gmail.com

ฯ Electronic mail: tucker.carrington@queensu.ca, Fax: 613-533-6669

** Electronic mail: berta.fernandez@usc.es
} 


\begin{abstract}
A new, highly accurate $a b$ initio ground-state intermolecular potential-energy surface (IPES) for the CO- $\mathrm{N}_{2}$ complex is presented. Thousands of interaction energies calculated with the CCSD(T) method and Dunning's aug-cc-pVQZ basis set extended with midbond functions were fitted to an analytical function. The global minimum of the potential corresponds to the geometry characterized by $r_{0}=7.856 a_{0}, \theta_{1}=111.3^{\circ}, \theta_{2}=159.7^{\circ}$, and $\phi_{2}=0^{\circ}$ and has an energy of $-118.2 \mathrm{~cm}^{-1}$. The symmetry-adapted Lanczos algorithm was used to compute rovibrational energies (up to $J=20$ ) on the new IPES. The RMSE with respect to experiment was found to be on the order of $0.038 \mathrm{~cm}^{-1}$ which confirms the very high accuracy of the potential. This level of agreement is among the best reported in the literature for weakly bound systems and considerably improves on those of previously published potentials.
\end{abstract}




\section{INTRODUCTION}

From the theoretical point of view accurate studies of weakly bound complexes pose a challenge. Not only are sophisticated methods accounting for electron-correlation effects required, but also extended basis sets are essential in order to obtain the results that, on one hand, can be compared with highly accurate experimental data and, on the other, can be used to make predictions in cases where good quality experimental data are not available. Furthermore, to obtain reliable intermolecular potential energy surfaces (IPESs) for complexes with more than 2 intermolecular coordinates the evaluation of thousands of $a b$ initio interaction energies is inevitable. To compute an accurate spectrum one must, after obtaining the IPES, solve the ro-vibrational Schroedinger without making approximations.

The CO- $\mathrm{N}_{2}$ dimer is a simple van der Waals (vdW) complex in which the intermolecular forces between two isoelectronic molecules, carbon monoxide (barely polar) and nitrogen (nonpolar), are of significant interest from the experimental and theoretical points of view. This system is of great relevance in atmospheric chemistry since $\mathrm{N}_{2}$ is one of the primary atmospheric constituents and $\mathrm{CO}$ is an important gaseous pollutant. Precise measurements and theoretical predictions of the effects of pressure and temperature on the spectrum of CO perturbed by the dominant atmospheric species, $\mathrm{N}_{2}$ and $\mathrm{O}_{2}$, are indispensable for the interpretation of atmospheric absorption data.

Spectra of the CO- $\mathrm{N}_{2}$ complex were first reported in 1996, when infrared (IR) spectra in the region of the CO fundamental band $\left(2146 \mathrm{~cm}^{-1}\right)$ were recorded by Kawashima and Nishizawa [1] and by Xu and McKellar [2]. In their experimental work Xu and McKellar [2] recorded the slit-jet IR spectrum of the complex in the $4.7 \mu \mathrm{m}$ region of the CO stretching vibration. They obtained a very simple spectrum, that could be interpreted as that of a (fictitious) system formed by a CO molecule and a rare gas atom of a mass of 28 a.m.u., due to an almost free rotation of the $\mathrm{N}_{2}$ molecule in the complex. An effective intermolecular separation of $4.025 \AA$ was deduced. Unfortunately, the results did not provide information about the angular orientation of the $\mathrm{N}_{2}$ molecule within the complex. The authors pointed out the need for theoretical calculations in order to predict the energy levels for higher lying excited states.

Pure rotational spectra of $\mathrm{CO}-\mathrm{N}_{2}$ with the ${ }^{14} \mathrm{~N}$ and ${ }^{15} \mathrm{~N}$ isotopes were observed by Kawashima et al. [3]. The authors also showed that, regarding the orientation of the $\mathrm{N}_{2}$ 
subunit and van der Waals stretching force constant, the $\mathrm{CO}-\mathrm{N}_{2}$ dimer is similar to the Ar- $\mathrm{N}_{2}$ and $\mathrm{Kr}-\mathrm{N}_{2}$ complexes.

At the same time, $\mathrm{Xu}$ et al. [4] studied the microwave and millimeter wave transitions of the ortho $\mathrm{N}_{2}$ states of the complex. Nuclear quadrupole hyperfine splittings were resolved and analysed in order to acquire additional information on the angular anisotropy of the IPES and the rotational and ro-vibrational spectra of two additional $\left({ }^{13} \mathrm{C}\right.$ and $\left.{ }^{18} \mathrm{O}\right)$ isotopomers were measured, too. The authors confirmed a planar, approximately T-shaped structure for the ground state of the complex $(K=0)$, with $\mathrm{N}_{2}$ as the top, $\mathrm{CO}$ as the leg, and with the $\mathrm{O}$ atom on average closer to $\mathrm{N}_{2}$ than the $\mathrm{C}$ atom.

In a complementary investigation [5] the rotational and ro-vibrational spectra of the COpara $\mathrm{N}_{2}$ complex were measured using a microwave spectrometer in the frequency region from 4 to $26 \mathrm{GHz}$. The behaviour of the complex could be interpreted neither in terms of a semirigid rotor model nor in terms of a totally free internal rotor. Therefore, the authors used several semirigid geometries associated with different $K$ values. They confirmed, as in Ref. 4, a similar planar, approximately T-shaped structure for the ground state $(K=0)$ of the ortho $\mathrm{N}_{2}$ state of the complex with an intermolecular distance of $4.026 \AA$ calculated from the experimental rotational constants using a pseudodiatomic approximation. The authors pointed out that $\mathrm{N}_{2}$ undergoes an almost free internal rotation within the complex. The geometry in the ortho $\mathrm{N}_{2}$ lower $K=1$ state is also T-shaped with $\mathrm{N}_{2}$ forming the leg and $\mathrm{CO}$ the top. The two investigated $\operatorname{paraN}_{2} K=1$ states correspond to the $\mathrm{N}_{2}$ molecule in a more nearly perpendicular orientation with respect to the intermolecular axis, whereas in the $K=0$ stack of the first excited bending state the $\mathrm{N}_{2}$ is located parallel to this axis. The values of the intermolecular distances were also reported for these states.

The IR spectrum of CO- $\mathrm{N}_{2}$ was further studied using a pulsed supersonic slit-jet and a rapid-scan tunable diode laser [6]. In the case of the $\mathrm{CO}-$ para $\mathrm{N}_{2}$ spin modification, the previous tentative assignment of just one subband $[1,2]$ was extended to include over 10 linked subbands in terms of three ground $\left(v_{\mathrm{CO}}=0\right)$ state stacks of levels (with $K=0$ and 1 ), and 7 excited state $\left(v_{\mathrm{CO}}=1\right)$ stacks (with $K=0,1$ and 2 ). In the case of the more abundant form, CO-ortho $\mathrm{N}_{2}$, an excited bending state was observed for the first time for both $v_{\mathrm{CO}}=0$ and 1 .

The first observation of the bending vibration of the CO- $\mathrm{N}_{2}$ complex in the millimeter wave range from 130 to $155 \mathrm{GHz}$ was reported in Ref. 7, providing precise information 
about the vibrational frequency and the rotational constants for this state. Six transitions, $P(2), P(1), R(0), R(1), R(2)$, and $R(3)$, associated with the ground and bending state $K=0$ levels of the ortho $\mathrm{N}_{2}$ spin modification, were measured and analysed.

In 2006, Surin et al. [8] carried out new measurements of the CO- $\mathrm{N}_{2}$ rotational transitions, and in 2015 the studies were completed by reporting new milimeter-wave transitions [9]. The authors concluded that in the $K=0$ state $\mathrm{CO}-$ para $_{2}$ has a geometry similar to that in the lower $K=1$ state of $\mathrm{CO}$-ortho $\mathrm{N}_{2}$, with $\mathrm{N}_{2}$ as the leg and $\mathrm{CO}$ as the top of the T-shaped complex, while in the $K=2$ state $\mathrm{N}_{2}$ forms the top and $\mathrm{CO}$ - the leg. The authors pointed out the necessity of theoretical research as an essential tool in the interpretation of the already available highly accurate experimental data.

In a recent paper [10], an analysis of a new broad-band $\left(2135-2165 \mathrm{~cm}^{-1}\right)$ spectrum of $\mathrm{CO}-\mathrm{N}_{2}$ obtained using a tunable continuous-wave quantum-cascade laser and a pulsed supersonic slit-jet source was presented. Almost 100 new rovibrational levels were assigned to nine new stacks, of which four are in the excited state of CO-ortho $\mathrm{N}_{2}$, three in the excited state of CO-para $\mathrm{N}_{2}$, and two in the ground state $\left(\nu_{\mathrm{CO}}=0\right)$ of CO-para $\mathrm{N}_{2}$. The calculated rotational constants for the various rotational level stacks varied from 0.0638 to $0.0768 \mathrm{~cm}^{-1}$ which corresponds to a range of 4.34 to $3.96 \AA$ for the effective intermolecular center-of-mass distance. However, using these data the authors could not resolve the CO-N 2 structure unambiguously and concluded that dynamical calculations of rotational energy levels are necessary.

The most important theoretical studies on the complex are the most recent. Fišer and Polák [11] investigated 11 structures of the complex using the coupled-cluster singles and doubles including connected triple corrections $[\mathrm{CCSD}(\mathrm{T})]$ and Møller-Plesset perturbation theory up to fourth order (MP4) methods and Dunning's correlation consistent augmented polarized valence aug-cc-pVXZ (X= D, T, Q) bases extended with a set of $3 s 3 p 2 d 1 f 1 g$ midbond functions. These results were further complemented with some new data [12]. The authors concluded that the results of the calculations support a considerable nonrigidity of the complex and the presence of multiple, nearly equally deep minima on the IPES, separated by barriers of about $1 \mathrm{~cm}^{-1}$ height. The most stable structures were two distorted T-shaped configurations where the $\mathrm{N}$-atom is pointing towards the $\mathrm{C}$-O bond, with intermolecular distances of ca. $4.1 \AA$ and $\operatorname{CCSD}(\mathrm{T}) /$ aug-cc-pVQZ $+3 s 3 p 2 d 1 \mathrm{f} 1 \mathrm{~g}$ interaction energies of -118.2 and $-117.4 \mathrm{~cm}^{-1}$. The interaction energy value for the first configuration 
was already close to the estimated complete basis-set (CBS) limit of $118.5 \mathrm{~cm}^{-1}$ [12].

The $a b$ initio study of the $\left(\mathrm{N}_{2}\right)_{n} \mathrm{CO}_{m}(n=1-7 ; m=1-3)$ complexes at the MP2 and $\operatorname{CCSD}(\mathrm{T})$ levels of theory with the $6-311+\mathrm{G}^{*}$ basis set showed that the $\mathrm{CO}-\mathrm{N}_{2}$ dimer exists in the form of a series of structurally different but energetically almost equivalent isomers [13]. According to the calculations, the interconversion between the conformers proceeds with the barriers not exceeding $0.07 \mathrm{kcal} \mathrm{mol}^{-1}$. Therefore, the internal movement of the molecules in the CO- $\mathrm{N}_{2}$ system is practically free.

The first ab initio IPES obtained for CO- $\mathrm{N}_{2}$ was published by Karimi-Jafari et al. [14] in 2011. The authors calculated a four-dimensional potential employing the MP4 method with a basis set obtained from the aug-cc-pVQZ basis set of Dunning and coworkers [15] by removing all the $g$ and $f$ functions; this basis was further supplemented by a set of $3 s 3 p 2 d 1 f$ midbond functions $[16,17]$. In contrast to previous experimental results the authors found that the intermolecular potential is characterized by a single, distorted T-shaped minimum (and its symmetrically equivalent partners) with $\mathrm{CO}$ at the top and $\mathrm{N}_{2}$ on the leg of the $\mathrm{T}$, and an energy of $-123.1 \mathrm{~cm}^{-1}$. This structure is similar to that assigned to an excited state in Ref. 5. The T-shaped ground state structure reported in Ref. 5 is identified on the MP4 IPES as a saddle point [14]. In order to estimate the correlation method error, the authors compared their MP4 interaction energies with the CCSD $(\mathrm{T})$ energies calculated with Dunning's aug-cc-pVTZ basis set augmented with the $3 s 3 p 2 d 1 f$ set of midbond functions. The differences were about $5 \mathrm{~cm}^{-1}$ in the proximity of the global minimum but rose to 15 $\mathrm{cm}^{-1}$ for the linear orientations of the complex.

The CO- $\mathrm{N}_{2}$ complex still remains a very attractive object for both theoretical and experimental studies and only very recently two papers $[18,19]$ concerning this system have been published.

Liu et al. [18] generated a five-dimensional IPES which accounted for the stretching of the C-O bond. The IPES was calculated with the explicitly correlated CCSD(T)-F12b method and the aug-cc-pVQZ basis set. A global minimum with a well depth of $-117.417 \mathrm{~cm}^{-1}$ was found at $\theta_{1}=73.0^{\circ}, \theta_{2}=13.8^{\circ}, \phi=0.0^{\circ}, R=4.155 \AA$, corresponding to a distorted T-shaped structure with $\mathrm{N}_{2}$ pointing towards the C-O bond. The authors reported RMSE discrepancies with respect to experimental data smaller than $0.068 \mathrm{~cm}^{-1}$ in the predicted IR transitions and intensities for the CO-ortho $\mathrm{N}_{2}$ complex as well as in the calculated energy levels for CO-para $\mathrm{N}_{2}$. Nonetheless, a considerable number of the misprints in the table with 
the energy levels makes this statement difficult to confirm.

The second ab initio calculated four-dimensional IPES has been reported by Surin et al. [19]. The calculations were carried out using the CCSD(T) method and the aug-cc-pVQZ basis set supplemented with midbond functions. The found global minimum of the IPES corresponds to the structure of $\theta_{\mathrm{CO}}=109.4^{\circ}, \theta_{\mathrm{N}_{2}}=162.8^{\circ}$, and $\varphi=0^{\circ}$ and $R_{\mathrm{e}}=7.86 a_{0}$, and has an energy of $-117.35 \mathrm{~cm}^{-1}$. Also a new millimeter-wave survey for the CO- $\mathrm{N}_{2}$ complex in the frequency range of 110-145 GHz was performed and several transitions not previously observed were detected and assigned. The computed energy levels were compared with the available experimental data and the authors found good agreement with experiment for all detected stacks. However, they noted that a significant number of the detected lines could still not be assigned.

However, it seems that each of the three published potentials suffers from some limitations. The potential for CO-N $\mathrm{N}_{2}$ from Ref. 14 is the least reliable. First, the MP4 method is well-known for overestimating interaction energies. Second, the authors removed from the basis set a significant portion of the diffuse functions that are responsible for a correct description of the intermolecular interactions. Third, the number of grid points that the authors used in their calculations does not seem to be sufficient to properly reproduce the anisotropy of the interaction, especially in the regions of short intermolecular distances.

The explicitly correlated CCSD(T)-F12 method [20-23], used in the calculations of the second potential [18], is becoming quite popular since contributions to calculated molecular correlation energies coming from single and double excitations are well converged with relatively small orbital bases. However, it seems that in the case of weak intermolecular interactions the errors introduced by the a/b/* approximations in CCSD-F12 and by a nonexplicitly correlated treatment of perturbative triples (with or without scaling) can become crucial at this level of accuracy [24]. It was also shown that, on one hand, CCSD(T)-F12 methods tend to perform more poorly for larger basis sets, but on the other, addition of midbond sets significantly improves the F12 calculated interaction energies. The authors in Ref. 18 did not test convergence of their method neither using larger bases or employing midbond functions.

The third potential [19] was obtained using the conventional $\operatorname{CCSD}(\mathrm{T})$ method and the aug-cc-pVQZ basis set further extended with a set of $3 s 2 p 1 d$ midbond functions; however, remarkable discrepancies between the calculated and experimental rotational states were 
still observed.

In this work we present a new four-dimensional interaction potential that seems to overcome all the above-mentioned deficiencies. The interaction energies were calculated at the CCSD(T) level, with the aug-cc-pVQZ basis set further extended with a set of $3 s 3 p 2 d 1 f 1 g$ midbond functions. For several representative geometries of the system we confirmed that the employed number of grid points is sufficient to correctly reproduce the anisotropy of the potential. Next, the calculated $a b$ initio interaction energies were fitted to an analytic expression. Then, the IPES was employed in calculations of the intermolecular states. The agreement between the calculated and experimental states is remarkably better than that reported for both of the most recent potentials $[18,19]$.

The manuscript is organized as follows: In Sec. II the details of the quantum-chemical calculations are given, then, in Sec. II B the results of the basis set studies are presented. In Sec. III we describe the analytical fit of the obtained IPES and some of its features, and in Sec. IV the details of the intermolecular-states studies are reported. Finally, in Sec. V we summarize and conclude.

\section{QUANTUM-CHEMICAL CALCULATIONS OF THE IPES}

\section{A. Computational details}

The coordinates describing the geometries used in the calculations are as follows. $r_{0}$ is the intermolecular distance between the centers of mass of the monomers, $\theta_{1}$ and $\theta_{2}$ are the angles between each of the molecular axis ( 1 for $\mathrm{CO}$ and 2 for $\mathrm{N}_{2}$ ) and the intermolecular axis, and $\phi_{2}$ is the difference between the azimuthal angles. For $\theta_{2}$ set to $180.0^{\circ}$ (or $0.0^{\circ}$ ) the values of $\theta_{1}=0.0$ and $180.0^{\circ}$ correspond to the linear CO $\cdots \mathrm{N}_{2}$ and $\mathrm{OC} \cdots \mathrm{N}_{2}$ geometries, respectively. In all calculations we fix the $\mathrm{CO}$ and $\mathrm{N}_{2}$ interatomic distances to the experimental $1.128323 \AA$ and $1.09768 \AA$ values [25], respectively. The intermolecular coordinates are depicted in Fig. 1.

Due to the good performance of the methodology employed in our previous studies on accurate calculation of IPESs of other vdW systems (Refs. 26, 27 and references cited therein), the interaction energies are corrected for the basis set superposition error (BSSE) using the counterpoise method [28] and are evaluated at the $\operatorname{CCSD}(\mathrm{T})$ level with the MOLPRO program [29]. In basis set completeness studies, we employed Dunning's doubly augmented 
correlation consistent basis sets, i.e. $\mathrm{x}$-aug-cc-pVXZ $(\mathrm{x}=-\mathrm{d}$; $\mathrm{X}=\mathrm{D}, \mathrm{T}, \mathrm{Q}, 5$; in the following denoted as xaXZ), as well as the core-valence aug-cc-pCVXZ (X=T, Q; denoted here as aCXZ) basis sets. These bases were extended with a set of $3 s 3 p 2 d 1 f 1 g$ midbond functions (denoted as 33211) with exponents of $0.90,0.30$, and 0.10 for the $s$ and the $p$ functions, 0.60 and 0.20 for the $d$ functions, and 0.30 for the $f$ and $g$ functions, that are placed in the middle of the van der Waals bond [30-32]. Relativistic effects were assessed using the second order Douglas-Kroll-Hess Hamiltonian as implemented within the MOLPRO program [29]

\section{B. Basis set study}

The results of the basis set study are presented in Tab. I. We chose 13 geometries with $r_{0}=4.4980 \AA\left(8.5 a_{0}\right), \theta_{1}=\theta_{2}$, and $\phi_{2}=0.0^{\circ}$. This slice cuts the IPES in the proximity of the global minimum of the system and allow us to compare the results with those reported in Ref. 14.

The overall shape of the potential slices is in qualitative agreement with that drawn in Fig. 1 of Ref. 14. As expected, the interaction energy increases (in absolute value) going from the aDZ to a5Z basis set. The convergence is faster with addition of midbond functions to the bases. However, the aQZ+33211 slice is slightly shallower than the aTZ+33211 one (by ca. $0.8 \mathrm{~cm}^{-1}$ for the lowest energy). This probably results from the fact that the slice does not pass directly through the global minimum and the topography of the IPES changes somewhat with basis set size. We further tested the influence of the frozen core approximation on the calculated interaction energies employing the core-valence aCXZ basis sets as well as the fully uncontracted ones. This effect is noticeable, but if we compare the results obtained within the frozen-core approximation with aQZ+33211 and those where all electrons were correlated with the aCQZ+33211 basis set for the geometry corresponding to the lowest interaction energy, the difference does not exceed $0.1 \mathrm{~cm}^{-1}$ (see Tab. I).

Further tests showed ( $c f$. Tab. I) that relativistic effects are negligible (significantly smaller than $0.01 \mathrm{~cm}^{-1}$ in the region of the global minimum) in the case of the $\mathrm{CO}-\mathrm{N}_{2}$ complex.

Since an appropriate choice of basis set balances the corresponding accuracy and the total cost, given the required number of geometries, we decided to carry out the calculations for the whole surface at the frozen core $\operatorname{CCSD}(\mathrm{T})$ level with the aQZ basis set augmented with 
the 33211 set of midbond functions.

\section{Choice of the number of grid points}

To choose correctly the number of grid points we perform some test calculations for different orientations of $\mathrm{CO}-\mathrm{N}_{2}$. Representative results for a region characterized by a pronounced anisotropy, are depicted in Fig 2. The coordinates of the chosen geometries are $r_{0}=3.70426 \AA\left(7.0 a_{0}\right), \theta_{1}=90^{\circ}$, and $\phi_{2}=0.0^{\circ}$, and the values of $\theta_{2}$ correspond to the abscissas of $n$-points Gauss-Lobatto quadrature. Here, we use the Gauss-Lobatto points since they include also points corresponding to 0 and 180 degrees unlike the Gauss-Legendre points employed in Ref. 14. The Gauss-Lobatto point distribution is similar to the distribution of the Legendre points used to compute the spectrum (see Sec. IV A). This ensures that the IPES is accurate where it is evaluated. The interaction energies are interpolated using $n=10, \ldots, 16$ nodes. The $a b$ initio calculated interaction energy values (black circles) employed in all the interpolations as well as some additionally calculated energies (red circles) are also presented in Fig 2. It is clear that the interpolated curves yield the correct overall shape of the exact curve and the accuracy increases with an increase of $n$. The exceptions are the regions where $\theta_{2}$ approaches 0 or 180 degrees, namely, in the proximities of the two T-shape geometries. The performance of the interpolations is worse for all the cases with an even $n$, mainly because they do not behave correctly at $\theta_{2}=0$ and 180 ( $c f$. Fig $2 \mathrm{a}$ and b). For the 10-point Gauss-Lobatto grid the differences between the interpolated and exact $a b$ initio values exceed $2 \mathrm{~cm}^{-1}$. The interpolations based on an odd number of points converge towards the correct shape of the curve; however, for this highly anisotropic orientation of the system such a convergence is slow. For other orientations of CO- $\mathrm{N}_{2}$ of lower anisotropy (not shown here) their performance is much more satisfactory.

Taking into account the above considerations, in the calculations of the IPES we decided to employ the 13-points Gauss-Lobatto grid for $\theta_{1}$ and $\theta_{2}$. This choice seems to be the optimal compromise between the desired accuracy and reliability and available computational resources. In most calculations we consider 12 intermolecular distances in $a_{0}$ : 5.0, 6.0, 7.0, 8.0, 9.0, 10.0, 11.0, 12.0, 14.0, 18.0, 23.0, 30.0. For some orientations we make additional calculations for distances of 4.0 and $40.0 a_{0}$. The values of $\phi_{2}$ correspond to the abscissas of 9-points Gauss-Lobatto quadrature. Because of the symmetry of the system $\theta_{1}$ and $\theta_{2}$ are 
varied between 0 and 180 degrees (13 grid points for each angle), while $\phi_{2}$ between 0 and 90 degrees (only 5 points are needed to be calculated for this angle). In total, the calculations are performed for slightly more than 10100 different geometries of the dimer.

\section{FITTING OF THE IPES}

Having determined the interaction energies for specific geometries via ab initio methods, we now turn to constructing an analytical model of the IPES. The analytical expression will be useful in other applications where a compact form of a potential is indispensable. Here, we model the IPES as a many body expansion, that is, as a function of the distances between the atoms in the complex.

The complex consists of two molecules $\mathrm{CO}$ and $\mathrm{N}_{2}$, the latter of which we consider made up of two atoms $\mathrm{N}$ and $\mathrm{N}^{\prime}$. Given a configuration of the complex, we can compute the following four distances

$$
\begin{aligned}
& d_{1}=d(C, N) \\
& d_{2}=d\left(C, N^{\prime}\right) \\
& d_{3}=d(O, N) \\
& d_{4}=d\left(O, N^{\prime}\right)
\end{aligned}
$$

Define the dimensionless quantities, $D_{i}$, by

$$
D_{i}=\exp \left(-a d_{i} / d_{\text {ref }}\right), \quad i=1,2,3,4,
$$

where the constant $d_{\text {ref }}=8 a_{0}$ is chosen to be the approximate distance between the two molecules, and the dimensionless constant $a$, the dilatation, is an adjustable parameter to be determined.

With four exponents $\left(e_{1}, e_{2}, e_{3}, e_{4}\right)$, we define the symmetrized term

$$
T_{\left(e_{1}, e_{2}, e_{3}, e_{4}\right)}=\frac{1}{2}\left(D_{1}^{e_{1}} D_{2}^{e_{2}} D_{3}^{e_{3}} D_{4}^{e_{4}}+D_{1}^{e_{2}} D_{2}^{e_{1}} D_{3}^{e_{4}} D_{4}^{e_{3}}\right) .
$$

Notice that if we exchange the positions of $\mathrm{N}$ and $\mathrm{N}^{\prime}$ (which does not change the shape of the complex), we interchange the value of $d_{1}$ and $d_{2}$ as well as that of $d_{3}$ and $d_{4}$. However, the value of the symmetrized term does not change. 
Our model of the interaction surface $V$ is a linear combination of symmetrized terms $[26$, $33,34]$, so that

$$
V=\sum_{\mathbf{e} \in E} c_{\mathbf{e}} T_{\mathbf{e}}
$$

where $E$ is the set of selected exponents, and we have written $\mathbf{e}=\left(e_{1}, e_{2}, e_{3}, e_{4}\right)$. So if we, for example, suppose that $E=\{(1,0,0,0),(2,1,0,1)\}$, we obtain

$$
V=c_{1000} T_{(1,0,0,0)}+c_{2101} T_{(2,1,0,1)}
$$

where we write e.g. $c_{1000}$ as a short hand for $c_{(1,0,0,0)}$. Now

$$
T_{(1,0,0,0)}=\frac{1}{2}\left(D_{1}^{1} D_{2}^{0} D_{3}^{0} D_{4}^{0}+D_{1}^{0} D_{2}^{1} D_{3}^{0} D_{4}^{0}\right)=\frac{1}{2}\left(D_{1}+D_{2}\right)
$$

and

$$
T_{(2,1,0,1)}=\frac{1}{2}\left(D_{1}^{2} D_{2}^{1} D_{3}^{0} D_{4}^{1}+D_{1}^{1} D_{2}^{2} D_{3}^{1} D_{4}^{0}\right)=\frac{1}{2}\left(D_{1}^{2} D_{2} D_{4}+D_{1} D_{2}^{2} D_{3}\right)
$$

Thus

$$
V=\frac{c_{1000}}{2}\left(D_{1}^{2} D_{2} D_{4}+D_{1} D_{2}^{2} D_{3}\right)+\frac{c_{2101}}{2}\left(D_{1}^{2} D_{2} D_{4}+D_{1} D_{2}^{2} D_{3}\right) .
$$

We pick 79 different symmetrized terms which gives us 80 adjustable parameters: the dilatation parameter $a$ on which the fitting function depends in a non-linear way, and the 79 coefficients, $c_{\mathbf{e}}$, defining the linear combination (i.e for fixed $a, V$ depends linearly on the last 79 parameters). Because we do not include the term with exponents $(0,0,0,0)$, the IPES will necessarily tend to zero when the distances tend towards infinity. We determined which symmetrized terms to use by heuristically searching for the set of 79 terms giving the best fit among all terms of degree less than or equal to 6 .

After determining the fit, we modified $V$ for small distances. This is to avoid physically unreasonable behavior when two atoms are close to each other, such as wildly oscillating interaction energies, or deeply negative energies. More precisely, we set

$$
V_{\text {mod }}=E_{\text {big }} \exp \left(-k d_{1} d_{2} d_{3} d_{4}\right)+V\left(1-\exp \left(-k d_{1} d_{2} d_{3} d_{4}\right)\right)
$$

where $k$ is some constant chosen by hand, and $E_{\text {big }}$ is some large energy. This means that $V_{\text {mod }}$ is equal to $E_{\mathrm{big}}$, when one of the distances $d_{i}$ is zero, and very close to $V$, when none of the distances $d_{1}, d_{2}, d_{3}$ and $d_{4}$ are very small. In practice, we used

$$
k=\left(\frac{1}{2 a_{0}}\right)^{4}
$$


and

$$
E_{\mathrm{big}}=10^{9} \mu E_{\mathrm{h}}
$$

We considered only geometries where the interaction energy did not exceed $100 \mu E_{\mathrm{h}}$ leaving us with 6710 points, and fitted the adjustable parameters to the $a b$ initio data. This gave us a good fit with an RMS deviation of $0.85 \mu E_{\mathrm{h}}\left(0.2 \mathrm{~cm}^{-1}\right)$, indicating that the model is accurate in the region relevant to describing the bound states. Of course, ignoring the regions where the interaction energy is high, means that there is no reason to expect that the model performs well there. And indeed, computing the deviation from all the ab initio interaction energies gives us an RMSE of $31000 \mu E_{\mathrm{h}}$.

The fitted value of the dimensionless dilation is $a=4.3799$, and the rest of the parameters are given in Tab. II. From the first table entry, we see that $c_{(0,0,1,0)}=-32.12 \mu E_{\mathrm{h}}$, so the first term in the sum giving $V$ is

$$
\begin{gathered}
-32.12 \mu E_{\mathrm{h}} T_{(0,0,1,0)}=\frac{-32.12 \mu E_{\mathrm{h}}}{2}\left(D_{1}^{0} D_{2}^{0} D_{3}^{1} D_{4}^{0}+D_{1}^{0} D_{2}^{0} D_{3}^{0} D_{4}^{1}\right) \\
=\frac{-32.12 \mu E_{\mathrm{h}}}{2}\left(D_{3}+D_{4}\right) .
\end{gathered}
$$

Using the analytical expression for the IPES, we find the global minimum to be $-538.47 \mu E_{\mathrm{h}}\left(-118.2 \mathrm{~cm}^{-1}\right)$, located at $r_{0}=7.856 a_{0}, \theta_{1}=111.34^{\circ}, \theta_{2}=159.70^{\circ}$, and $\phi_{2}=0^{\circ}$. A view of the IPES for $r_{0}=7.856 a_{0}$ and $\phi_{2}=0^{\circ}$ is depicted in Fig. 3. The minimal potential-energy configurations (all equal by symmetry) are marked with blue dots. A nearly barrierless disrotatory path permitting a floppy large-amplitude motion of the complex is observed. This result is similar to that found for the CO dimer [35].

The global minimum found in this work is by ca. $0.8 \mathrm{~cm}^{-1}$ deeper than those reported previously $[18,19]$. The corresponding intermolecular distance is similar for all the three potentials (ca. 7.85-7.86 $a_{0}$ ), while our $\theta_{1}$ is slightly larger and $\theta_{2}$ slightly smaller than those from previous studies $[18,19]$. To further check the performance of the analytical fit, we evaluated some additional CCSD(T)/aug-cc-pVQZ-33211 interaction energies in the vicinity of the expected global minimum on a grid of points. The lowest interaction energy of $-118.04 \mathrm{~cm}^{-1}$ was found at $r_{0}=7.8 a_{0}, \theta_{1}=109.0^{\circ}, \theta_{2}=162.0^{\circ}$, and $\phi_{2}=0^{\circ}$. This result is in concordance with that obtained from the fit. 


\section{INTERMOLECULAR STATES}

\section{A. Variational calculations}

The rovibrational levels of $\mathrm{CO}-\mathrm{N}_{2}$ were calculated using a variational method called DSL $[36,37]$ which uses a product basis with discrete variable representation (DVR) functions (D) [38] for the stretches and spherical harmonic type functions (S) for the bends and a symmetry adapted Lanczos eigensolver (L). The bend basis is appropriate for dealing with large amplitude bending motions. The calculation is carried out with the RV4 code [39] that implements the DSL method. It uses the polyspherical coordinates $\left(r_{1}, r_{2}, r_{0}, \theta_{1}, \theta_{2}\right.$, $\left.\phi_{2}\right)$ associated with vector $\mathbf{r}_{\mathbf{1}}$ (for $\mathrm{CO}$ ), vector $\mathbf{r}_{\mathbf{2}}$ (for $\mathrm{N}_{2}$ ), and the Jacobi vector $\mathbf{r}_{\mathbf{0}}$, shown in Fig. 1. Because the intramonomer vibrational frequencies are much higher than their intermonomer counterparts, it is justified to fix $r_{1}$ and $r_{2}$ to their respective ground state values. In the DSL method, potential energy integrals are computed with Gauss quadrature. Eigenvalues and eigenvectors are determined with the symmetry-adapted Lanczos (SAL) algorithm [40, 41].

Since a thorough description of the DSL method applied to a similar system $\left(\mathrm{N}_{2} \mathrm{O}\right)_{2}$ is reported in Refs. 42, 43, only the computational details specific to the present study are given here. Each basis function is a product

$$
f_{\alpha_{0}}(R) u_{l_{1} l_{2} m_{2} K}^{J M P}\left(\theta_{1}, \theta_{2}, \phi_{2} ; \alpha, \beta, \gamma\right)
$$

where $f_{\alpha_{0}}(R)$ is a DVR function for the stretch coordinate and $u_{l_{1} l_{2} m_{2} K}^{J M P}$ is the parity adapted bend-rotation function. The latter was expressed as a linear combination of two products of an associated Legendre function for $\theta_{1}$, a spherical harmonic function for $\left(\theta_{2}, \phi_{2}\right)$, and a Wigner function for the Euler angles $(\alpha, \beta, \gamma)$, with $P=0$ and $P=1$ corresponding to even and odd parity, respectively.

In our calculation, we use the full permutation-inversion group of $\mathrm{CO}-\mathrm{N}_{2}, G_{4}$. It has four irreducible representations: $A+, B+, A-$, and $B-$. $A / B$ label levels that are symmetric/antisymmetric under permutation of the two $\mathrm{N}$ atoms. +/- label even/odd levels. In our calculation, the levels of the two nuclear spin species are obtained in different symmetry blocks. CO- ortho $\mathrm{N}_{2}$ levels appear in the $A$ symmetry block and CO-para $\mathrm{N}_{2}$ levels appear in the $B$ symmetry block. The lowest level of $\mathrm{CO}$ - ortho $\mathrm{N}_{2}$ is lower than the lowest level of CO-para $\mathrm{N}_{2}$ because the two levels are associated with $j_{\mathrm{N}_{2}}=0$ and 1 , respectively. 
Because of the large-amplitude motion and the resulting low-lying vibrational states, rather than using a traditional asymmetric rotor Hamiltonian to fit the levels, spectroscopists group the rovibrational levels into stacks and fit the levels in each stack. A stack is labelled by $\left(j_{\mathrm{CO}}, j_{\mathrm{N}_{2}} ; K, \sigma\right)$, where $\sigma=e / f$ corresponds to $(-1)^{J+P}= \pm 1$. Each stack of levels is fitted with a simple rotational energy level expression based on a linear rotor Hamiltonian with a doubling term that splits the $e / f$ components of $K>0$ stacks. See Eq. (1) of Ref. 2 for $\mathrm{CO}-\mathrm{N}_{2}$, which has been adopted in all subsequent $\mathrm{CO}-\mathrm{N}_{2}$ papers cited in this work. In our calculations, $K$ can be unambiguously assigned by analysing the wavefunctions [35]. $j_{\mathrm{CO}}, j_{\mathrm{N}_{2}}$ are simply obtained by matching computed and experimental levels.

The parameters for computing the intermolecular rovibrational levels using the RV4 code are all given in Tab. III. The choice of the basis functions for $r_{0}$ merits further discussion. Usually a potential-optimized discrete variable representation (PODVR) basis $[44,45]$ is a good choice. The PODVR basis is efficient if a good reference potential, usually either a cut potential or a minimized potential, can be found. It has the advantage of being black-box because there are no parameters to adjust. For $\mathrm{CO}-\mathrm{N}_{2}$, bases obtained from the cut and minimized potentials are both poor, due to coupling. Owing to the fact that neither the cut nor the minimized PODVR basis is good, we use a tri-diagonal Morse (TDM) DVR basis [44]. However, it takes more effort to optimize the four parameters of the TDM DVR basis: $D_{\mathrm{e}}$, $\omega_{\mathrm{e}}, r_{\mathrm{e}}$ and $\alpha$. The first three parameters are optimized by comparing a few of the lowest $J=0$ levels computed with a TDM basis and a set of benchmark levels computed using 120 sine DVR basis. Our final TDM DVR basis parameters are $D_{\mathrm{e}}=108 \mathrm{~cm}^{-1}, \omega_{\mathrm{e}}=18$ $\mathrm{cm}^{-1}$, and $r_{\mathrm{e}}=8.45 a_{0}$. The fourth TDM DVR basis parameter $\alpha$ depends on the other parameters ( $\alpha$ appears in the associated Laguerre polynomial $L_{n}^{\alpha}$ ). As discussed in Ref. 46, we follow Wei and Carrington's choice [44], $\alpha=A-2[A / 2]=0$ with $A=4 D_{\mathrm{e}} / \omega_{\mathrm{e}}=24$ so that all the bound states of the Morse Hamiltonian are exactly reproduced by the TDM basis (the Morse Hamiltonian that corresponds to these parameters has $[\mathrm{A} / 2]=12$ bound states, fewer than the number of basis functions, $\left.N_{r_{0}}=30\right)$.

\section{B. Existing experimental data}

We compare the experimental rovibrational levels on the $v_{\mathrm{CO}}=0$ ground state of the two spin species with our calculated levels. The experimental levels are collected from the 
millimeter-wave (MMW) study by Surin et al. [8] and the IR study by Rezaei et al. [10]. There are 3 stacks of levels for CO-ortho $\mathrm{N}_{2}$. They are, in the order of increasing energy, $(0,0,0 e),(1,0,1 e / f)$, and $(1,0,0 e)$ and are labelled as stacks $a, b, c$. There are 5 stacks of levels for CO-para $\mathrm{N}_{2}$. They are, in the order of increasing energy, $(0,1,0 e),(0,1,1 e / f)$, $(1,1,1 e / f),(1,1,0 e)$, and $(1,1,2 e / f)$ and are labelled as stacks $a, b, c, d, e$ (see Tab. IV). Note that a correction term $0.0048 \mathrm{~cm}^{-1}$ needs to be added to the $(0,1,1 f)$ levels of COpara $\mathrm{N}_{2}$ obtained from the MMW study (Tab. 8 of Ref. 8), which was first pointed out by Rezaei et al. in their IR study [10]. This is because the $(0,1,1 f)$ stack was not linked to other stacks in the MMW experiment and the band center of this stack was "floated" or corrected by Rezaei et al.. In the IR experiment of Rezaei et al. [10], the $(0,1,1 f)$ stack is linked with the $(0,1,0 e)$ stack via transitions to the common upper state $(1,1,0 e)$ of the $v_{\mathrm{CO}}=1$ excited state. We note that this correction is confirmed by a new MMW experiment [9] where the $(0,1,1 f)$ stack is again linked with the $(0,1,0 e)$ via transitions to the common upper state $(0,1,0 e)$.

\section{Theoretical results}

Even and odd levels for both CO-ortho $\mathrm{N}_{2}$ and CO-para $\mathrm{N}_{2}$ were computed for each $J$ up to $J=20$ and the complete set can be obtained from the authors on request. Levels up to $J=10$ with experimental assignments are given in Tab. $\mathrm{V}$ for $\mathrm{CO}$-ortho $\mathrm{N}_{2}$. Levels up to $J=8$ with experimental assignments are given in Tab. VI for CO-para $\mathrm{N}_{2}$. A graphical comparison of the experimental and calculated intermolecular levels for CO-ortho $\mathrm{N}_{2}$ and CO-para $\mathrm{N}_{2}$ is presented in Figs. 4a and b, respectively. The agreement between theory and experiment is remarkably good, making assignments straightforward and unambiguous. They were also facilitated by knowing the symmetry of each of the computed levels, owing to the use of the symmetry adapted Lanczos procedure and by assigning $K$ to each level using the wavefunctions.

Interestingly the agreement is significantly better for the CO-ortho $\mathrm{N}_{2}$ levels, with an RMSE over 41 levels of just $0.017 \mathrm{~cm}^{-1}$. Those errors are both positive and negative with an average close to zero. For the CO-para $\mathrm{N}_{2}$ levels, the errors are larger (the RMSE for 40 levels is $0.045 \mathrm{~cm}^{-1}$ ) and more systematic, as the computed levels are below the experimental ones in all cases. The level of agreement obtained in these results is among the best reported 
in the literature for vdWs systems. It could be of interest to explore this further in terms of the hierarchy of electronic structure methods employed (also going beyond the CCSD(T) approximation). It remains to be determined whether various terms in high-level composite energies [47] for this system would be found to be negligible or if the currently adopted procedure benefits from particularly favourable cancellation of errors.

Variational calculations on ab initio IPESs seldom achieve accuracy as good as $1 \mathrm{~cm}^{-1}$ and accuracy better than $0.1 \mathrm{~cm}^{-1}$ is very rare. The lowest bending vibrational energy level has been observed for both spin species: $4.666 \mathrm{~cm}^{-1}$ for CO-ortho $\mathrm{N}_{2}$ and $4.496 \mathrm{~cm}^{-1}$ for CO-para $\mathrm{N}_{2}$. These two vibrational levels are linked by a red arrow in Fig. 4. For these two vibrational levels, the calculated minus observed differences are only $0.02 \mathrm{~cm}^{-1}$ and 0.07 $\mathrm{cm}^{-1}$, respectively. It is rare to find this sort of accuracy for a vdW system. Much of the overall RMSE for the two spin species can be traced to the vibrational errors. Errors in differences between $J$ levels for a particular stack are typically on the order of $0.001 \mathrm{~cm}^{-1}$ and never larger than $0.01 \mathrm{~cm}^{-1}$ (see e.g. Fig. 4). Errors in differences of rotational levels are smaller than for the correspnding vibrational levels for many molecules, because the structure of the molecule predicted by ab initio theory is usually more accurate than the shape of the IPES on which the vibrational levels depend.

The lowest two stacks, stack $a(K=0)$ and $b(K=1)$ of $\mathrm{CO}$-para $\mathrm{N}_{2}$ are closely spaced and strong Coriolis interaction between them was observed experimentally [5]. After analysing the wavefunctions, we have also found that some levels in these two stacks have two dominant $K$ components. These levels are labelled as 01 or 10 in the $K$ column of Tab. VI. 01 (10) means the $K=0(K=1)$ contribution is slightly larger than the $K=1(K=0)$ contribution (see Tab. VI). The same coupling was also discussed in the theoretical study of Surin et al. [19].

When compared to the results from the two most recent investigations [18, 19], our potential gives significantly better agreement with experimental data. In Ref. 18 the differences between the reported theoretical and the available experimental intermolecular energy levels are as large as $0.27 \mathrm{~cm}^{-1}$, while in Ref. 19 these differences are considerably smaller. Here we further improve the results for the CO- $\mathrm{N}_{2}$ complex, by evaluating a more accurate IPES, with the potential minimum close to the exact $\operatorname{CCSD}(\mathrm{T})$ result and with a better performance regarding the evaluation of the intermolecular states, lowering the RMSE from 0.1 to $0.038 \mathrm{~cm}^{-1}$. This is clearly seen in Fig. 4 where the theoretical levels of Ref. 19 are 
compared with our theoretical levels and the experimental levels.

\section{SUMMARY AND CONCLUSIONS}

An accurate IPES for the CO- $\mathrm{N}_{2}$ dimer was constructed using the high-level ab initio CCSD(T)/aug-cc-pVQZ+33211 method. More than 10000 calculated points were fitted to an analytical function. A symmetrized fitting approach yields an RMSE of $0.2 \mathrm{~cm}^{-1}$ representing the data in the low energy regions relevant to the rovibrational levels computed in this study. Using the analytical fit of the IPES, we have found the global minimum with an energy of $-538.47 \mu E_{\mathrm{h}}\left(-118.2 \mathrm{~cm}^{-1}\right)$ located at $r_{0}=7.856 a_{0}, \theta_{1}=111.34^{\circ}, \theta_{2}=159.70^{\circ}$, and $\phi_{2}=0^{\circ}$. The nominally T-shaped structures of the complex were confirmed, although a low-energy channel permitting floppy large-amplitude motion was observed.

The symmetry-adapted Lanczos method was used in highly converged rovibrational variational calculations. Stacks of rovibrational levels for both CO-ortho $\mathrm{N}_{2}$ and CO-para $\mathrm{N}_{2}$ were computed up to $J=20$. The accuracy of the IPES is remarkable, with an RMSE of only $0.038 \mathrm{~cm}^{-1}$ over 81 experimentally assigned levels. This new potential considerably improves those previously evaluated for the complex, in particular the most accurate available [19], providing exceptionally good agreement between the calculated and the experimental intermolecular states.

\section{Conflicts of interest}

There are no conflicts of interest to declare.

\section{Acknowledgments}

Research financed by the National Science Centre in Poland within the OPUS 8 project No. 2014/15/B/ST4/04551. The research is a part of the program of the National Laboratory FAMO in Torun, Poland. This work was supported by the Spanish Ministerio de Ciencia e Innovación (CTQ2011-29311-C02-01 project) and the Xunta de Galicia (GRC GI-1905 project). The authors acknowledge the computational grant G51-15 from the Interdisciplinary Centre of Mathematical and Computer Modelling (ICM) of the University of Warsaw. Calculations have been carried out using resources provided by Wroclaw Centre 
for Networking and Supercomputing (http://wcss.pl), Grant No. 294. Centro de Supercomputación de Galicia (CESGA) is acknowledged for computational resources. RD is supported by the US National Science Foundation (No. CHE-1566246) and TC by the Canadian Natural Sciences and Engineering Research Council. 
[1] Y. Kawashima and K. Nishiza, Pulsed molecular beam infrared absorption spectroscopy of the $\mathrm{N}_{2}$-CO complex, Chem. Phys. Lett. 249, 87 (1996).

[2] Y. Xu and A. R. W. McKellar, Continuous slit-jet infrared spectrum of the CO-N $\mathrm{N}_{2}$ complex, J. Chem. Phys. 104, 2488 (1996).

[3] Y. Kawashima, Y. Ohshima, and Y. Endo, Rotational spectrum of the $\mathrm{N}_{2} \cdots \mathrm{CO}$ complex: ortho- $\mathrm{N}_{2}$ and para- $\mathrm{N}_{2}$, Chem. Phys. Lett. 315, 201 (1999).

[4] Y. Xu, W. Jäger, L. A. Surin, I. Pak, L. A. Panfilov, and G. Winnewisser, Microwave and millimeter wave study of ortho- $\mathrm{N}_{2}$ states of CO- $\mathrm{N}_{2}$, J. Chem. Phys. 111, 10476-10483 (1999).

[5] Y. Xu and W. Jäger, The dynamics of the CO- $\mathrm{N}_{2}$ interaction: Strong Coriolis coupling in CO-paraN 2 , J. Chem. Phys. 113, 514-524 (2000).

[6] C. Xia, A. R. W. McKellar, and Y. Xu, Infrared spectrum of the CO- $\mathrm{N}_{2}$ van der Waals complex: Assignments for CO-para $\mathrm{N}_{2}$ and observation of a bending state for CO-ortho $\mathrm{N}_{2}$, J. Chem. Phys. 113, 525 (2000).

[7] L. A. Surin, H. S. P Müller, E. V. Alieva, B. S. Dumesh, G. Winnewisser, and I. Pak, Detection of the bending vibration of the CO-ortho $\mathrm{N}_{2}$ complex, J. Mol. Struct. 612, 207 (2002).

[8] L. A. Surin, A. V. Potapov, H. S. P. Müller, V. A. Panfilov, B. S. Dumesh, T. F. Giesen, and S. Schlemmer, Millimeter-wave study of the CO- $\mathrm{N}_{2}$ van der Waals complex: new measurements of CO-ortho- $\mathrm{N}_{2}$ and assignments of new states of $\mathrm{CO}-$ para- $\mathrm{N}_{2}$, J. Mol. Struct. 795, 198 (2006).

[9] L. A. Surin, A. Potapov, H. S. P. Müller, and S. Schlemmer, A new millimeter-wave observation of the weakly bound CO-N 2 complex, J. Mol. Spectrosc. 307, 54 (2015).

[10] M. Rezaei, K. H. Michaelian, N. Moazzen-Ahmadi, and A. R. W. McKellar, A new look at the infrared spectrum of the weakly bound $\mathrm{CO}-\mathrm{N}_{2}$ complex, J. Phys. Chem. A 117, 13752 (2013).

[11] J. Fišer and R. Polák, An ab initio study of the CO-N $\mathrm{N}_{2}$ complex, Chem. Phys. Lett. 360, 565 (2002).

[12] J. Fišer, T. Boublík, and R. Polák, Combining rule for interaction energies of the $(\mathrm{CO})_{2}$, $\left(\mathrm{N}_{2}\right)_{2}$ and CO-N $\mathrm{N}_{2}$ complexes, Mol. Phys. 101, 3409 (2003).

[13] T. N. Gribanova, A. A. Milov, O. A. Gapurenko, A. G. Starikov, V. A. Gurashvili, R. M. 
Minyaev, and V. I. Minkin, Structure and stability of the mixed polymolecular complexes of nitrogen and carbon nonooxide: A quantum chemical study, Russ. J. Gen. Chem. 81, 807 (2011).

[14] M. H. Karimi-Jafari, A. Maghari, and A. Farjamnia, Intermolecular potential energy surface of the $\mathrm{N}_{2}$-CO dimer: Ab initio investigation and analytical representation, J. Phys. Chem. A 115, 1143 (2011).

[15] T. H. Dunning, Jr., Gaussian basis sets for use in correlated molecular calculations. I. The atoms boron through neon and hydrogen, J. Chem. Phys. 90, 1007 (1989).

[16] F.-M. Tao and Y.-K. Pan, Ab initio potential energy curves and binding energies of $\mathrm{Ar}_{2}$ and $\mathrm{Mg}_{2}$, Mol. Phys. 81, 507 (1994).

[17] F.-M. Tao and W. Klemperer, Accurate ab initio potential energy surfaces of Ar-HF, Ar- $\mathrm{H}_{2} \mathrm{O}$, and $\mathrm{Ar}-\mathrm{NH}_{3}$, J. Chem. Phys. 101, 1129 (1994).

[18] J.-M. Liu, Y. Zhai, X.-L. Zhang, and H. Li, Intermolecular configurations dominated by quadrupole-quadrupole electrostatic interactions: explicit correlation treatment of the fivedimensional potential energy surface and infrared spectra for the $\mathrm{CO}-\mathrm{N}_{2}$ complex, Phys. Chem. Chem. Phys. 20, 2036 (2018).

[19] L. A. Surin, I. V. Tarabukin, S. Schlemmer, Y. N. Kalugina, and A. van der Avoird, Ab initio potential and rotational spectra of the CO-N 2 complex, J. Chem. Phys. 148, 044313 (2018).

[20] H. Fliegl, W. Klopper, and C. Hättig, Coupled-cluster theory with simplified linear-r12 corrections: The CCSD(R12) model, J. Chem. Phys. 122, 084107 (2005).

[21] H. Fliegl, C. Hättig, and W. Klopper, Inclusion of the (T) triples correction into the linear-r12 corrected coupled-cluster model CCSD(R12), Int. J. Quantum Chem. 106, 2306 (2006).

[22] T. B. Adler, G. Knizia, and H.-J. Werner, A simple and efficient CCSD(T)-F12 approximation, J. Chem. Phys. 127, 221106 (2007).

[23] C. Hättig, D. P. Tew, and A. Köhn, Communications: Accurate and efficient approximations to explicitly correlated coupled-cluster singles and doubles, CCSD-F12, J. Chem. Phys. 132, $231102(2010)$.

[24] K. Patkowski, On the accuracy of explicitly correlated coupled-cluster interaction energies have orbital results been beaten yet?, J. Chem. Phys. 137, 034103 (2012).

[25] K. P. Huber and G. Herzberg, Molecular Spectra and Molecular Structure. IV. Constants of Diatomic Molecules, van Nostrand Reinhold Company New York (1979), see 
http://webbook.nist.gov.

[26] H. Cybulski, B. Fernández, C. Henriksen, and P. M. Felker, Ab initio ground state phenylacetylene-argon intermolecular potential energy surface and rovibrational spectrum, J. Chem. Phys. 137, 074305 (2012).

[27] C. R. Munteanu and B. Fernández, Accurate intermolecular ground-state potential-energy surfaces of the HCCH-He, Ne, and Ar van der Waals complexes, J. Chem. Phys. 123, 014309 (2005).

[28] S. F. Boys and F. Bernardi, The calculation of small molecular interactions by the differences of separate total energies. Some procedures with reduced errors, Mol. Phys. 19, 553 (1970).

[29] H.-J. Werner, P. J. Knowles, G. Knizia, F. R. Manby, M. Schütz, et al., MOLPRO, version 2010.1, a package of ab initio programs (2010), see: http://www.molpro.net.

[30] F.-M. Tao and Y.-K. Pan, Moller-Plesset perturbation investigation of the $\mathrm{He}_{2}$ potential and the role of midbond basis functions, J. Chem. Phys. 97, 4989 (1992).

[31] F.-M. Tao and Y.-K. Pan, An accurate ab initio calculation of the $\mathrm{Ne}_{2}$ potential, Chem. Phys. Lett. 194, 162 (1992).

[32] F.-M. Tao, The use of midbond functions for ab initio calculations of the asymmetric potentials of He-Ne and He-Ar, J. Chem. Phys. 98, 3049 (1993).

[33] Z. Xie and J. M. Bowman, Permutationally invariant polynomial basis for molecular energy surface fitting via monomial symmetrization, J. Chem. Theory Comput. 6, 26 (2010).

[34] B. Fernández, C. Henriksen, and D. Farrelly, Refined ab initio intermolecular ground-state potential energy surface for the $\mathrm{He}_{-} \mathrm{C}_{2} \mathrm{H}_{2}$ van der Waals complex, Mol. Phys. 111, 1173 (2013).

[35] R. Dawes, X. Wang, and T. Carrington Jr., CO dimer: New potential energy surface and rovibrational calculations, J. Phys. Chem. A 117, 7612 (2013).

[36] X.-G. Wang and T. Carrington Jr., New ideas for using contracted basis functions with a Lanczos eigensolver for computing vibrational spectra of molecules with four or more atoms, J. Chem. Phys. 117, 6923 (2002).

[37] X.-G. Wang, T. Carrington Jr., J. Tang, and A. R. W. McKellar, Theoretical and experimental studies of the infrared rovibrational spectrum of $\mathrm{He}_{2}-\mathrm{N}_{2} \mathrm{O}$, J. Chem. Phys. 123, 034301 (2005).

[38] J. C. Light and T. Carrington Jr, Discrete-variable representations and their utilization, Adv. Chem. Phys. 114, 263 (2007).

[39] X.-G. Wang and T. Carrington Jr., RV4 is a package of programs to compute rovibrational 
levels and wavefunctions of tetra-atomic molecules.

[40] R. Chen and H. Guo, A single Lanczos propagation method for calculating transition amplitudes. II. Modified QL and symmetry adaptation, J. Chem. Phys. 114, 1467 (2001).

[41] X.-G. Wang and T. Carrington Jr., A symmetry-adapted Lanczos method for calculating energy levels with different symmetries from a single set of iterations, J. Chem. Phys. 114, $1473(2001)$.

[42] R. Dawes, X.-G. Wang, A. W. Jasper, and T. Carrington Jr., Nitrous oxide dimer: A new potential energy surface and rovibrational spectrum of the nonpolar isomer, J. Chem. Phys. 133, $134304(2010)$.

[43] X.-G. Wang, T. Carrington Jr., R. Dawes, and A. W. Jasper, The vibration-rotation-tunneling spectrum of the polar and T-shaped-N-in isomers of $(\mathrm{NNO})_{2}$, J. Mol. Spectrosc. 268, 53 (2011)

[44] H. Wei and T. Carrington Jr., The discrete variable representation of a triatomic Hamiltonian in bond length-bond angle coordinates, J. Chem. Phys. 97, 3029 (1992).

[45] J. Echave and D. C. Clary, Potential optimized discrete variable representation, Chem. Phys. Lett. 190, 225 (1992).

[46] X.-G. Wang and T. Carrington Jr., The vibration-rotation-tunneling levels of $\mathrm{N}_{2}-\mathrm{H}_{2} \mathrm{O}$ and $\mathrm{N}_{2}-\mathrm{D}_{2} \mathrm{O}$, J. Chem. Phys. 143, 024303 (2015).

[47] R. Dawes and S. A. Ndengué, Single- and multireference electronic structure calculations for constructing potential energy surfaces, Int. Rev. Phys. Chem. 35, 441 (2016).

[48] A. W. Mantz, J. K. G. Watson, K. N. Rao, D. L. Albritton, A. L. Schmeltekopf, and R. N. Zare, Rydberg-Klein-Rees potential for the $\mathrm{X}^{1} \Sigma^{+}$state of the CO molecule, J. Mol. Spectrosc. 39, 180 (1971).

[49] J. M. Hutson, BOUND computer code, version 16, distributed by Collaborative Computational Project No. 6 of the Science and Engineering Research Council (UK) (2009). 
FIG. 1: The intermolecular coordinates $r_{0}, \theta_{1}, \theta_{2}$ and $\phi_{2}$

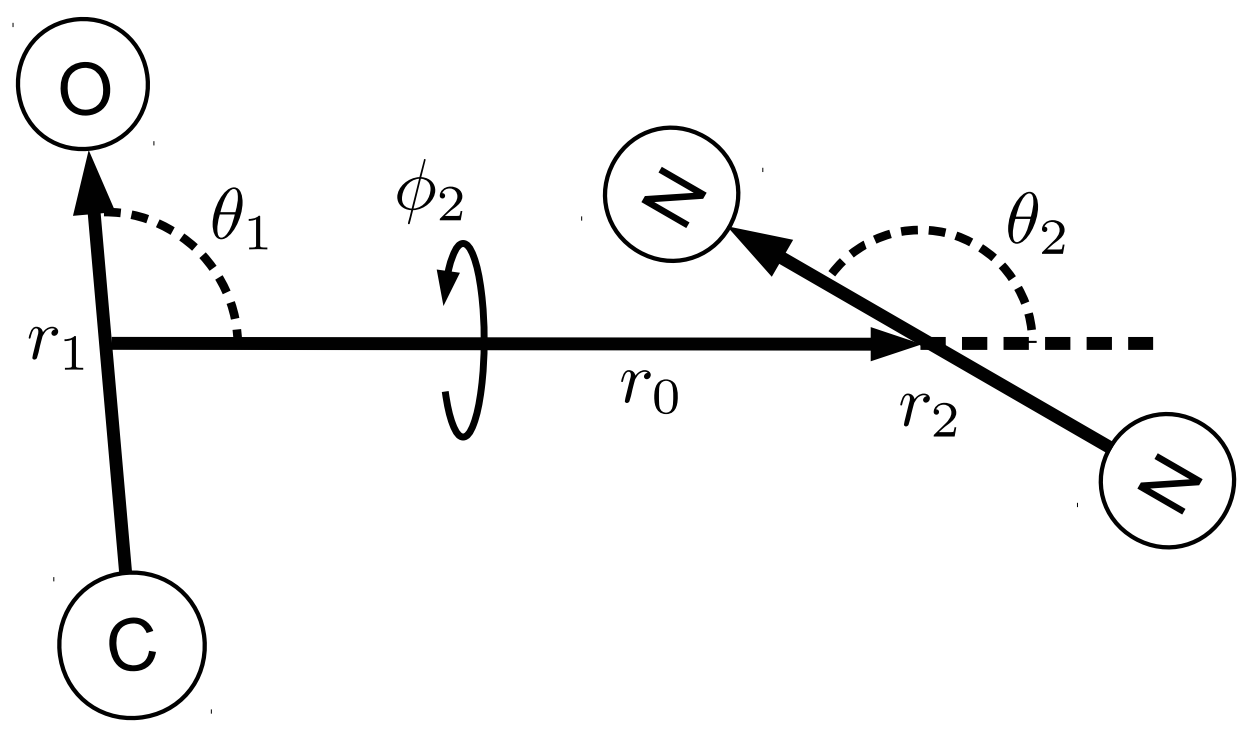



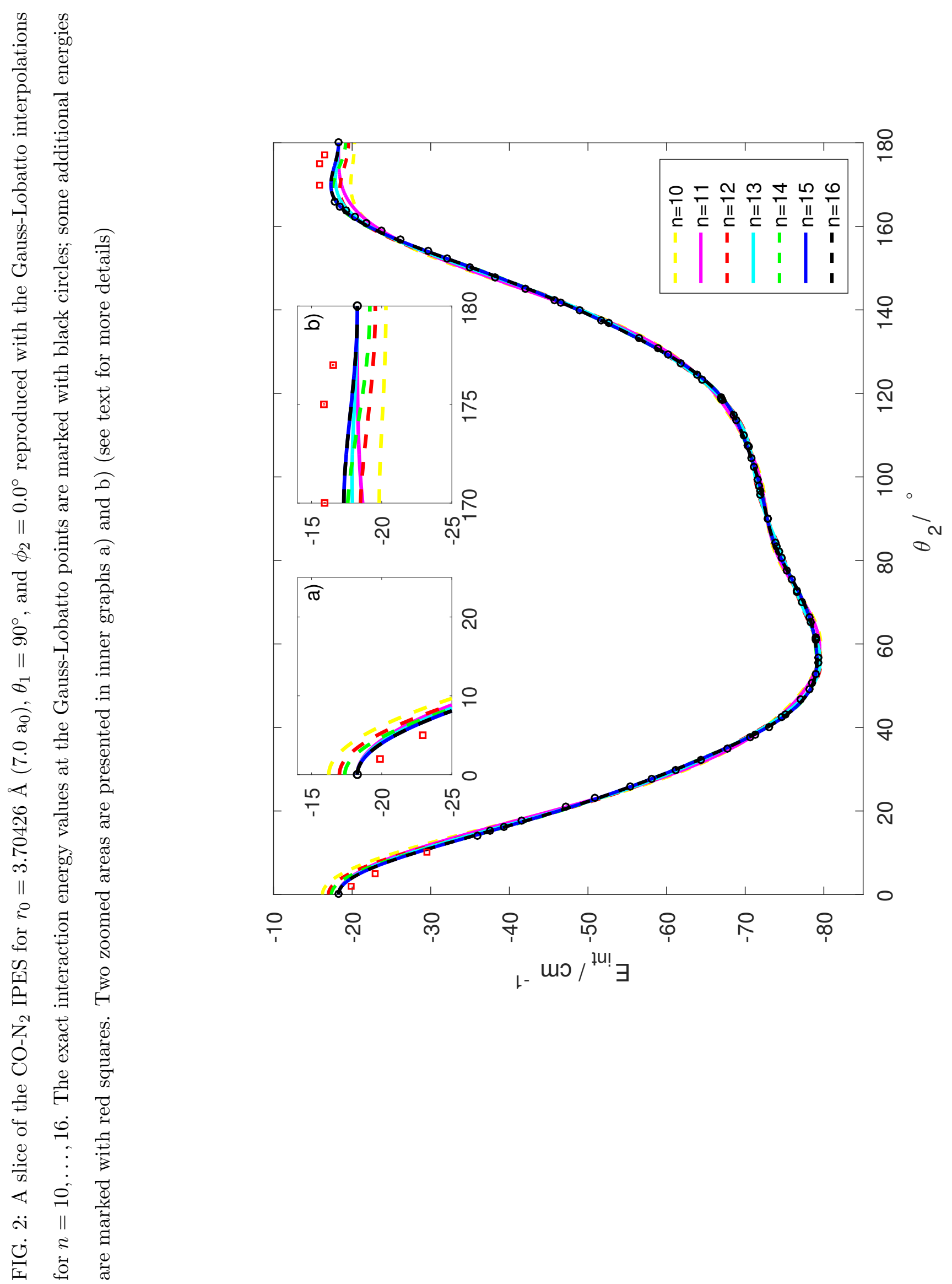
FIG. 3: CO- $\mathrm{N}_{2}$ IPES for $r_{0}=7.856 a_{0}$ and $\phi_{2}=0^{\circ}$. The minimal potential energy configuration is marked with blue dots
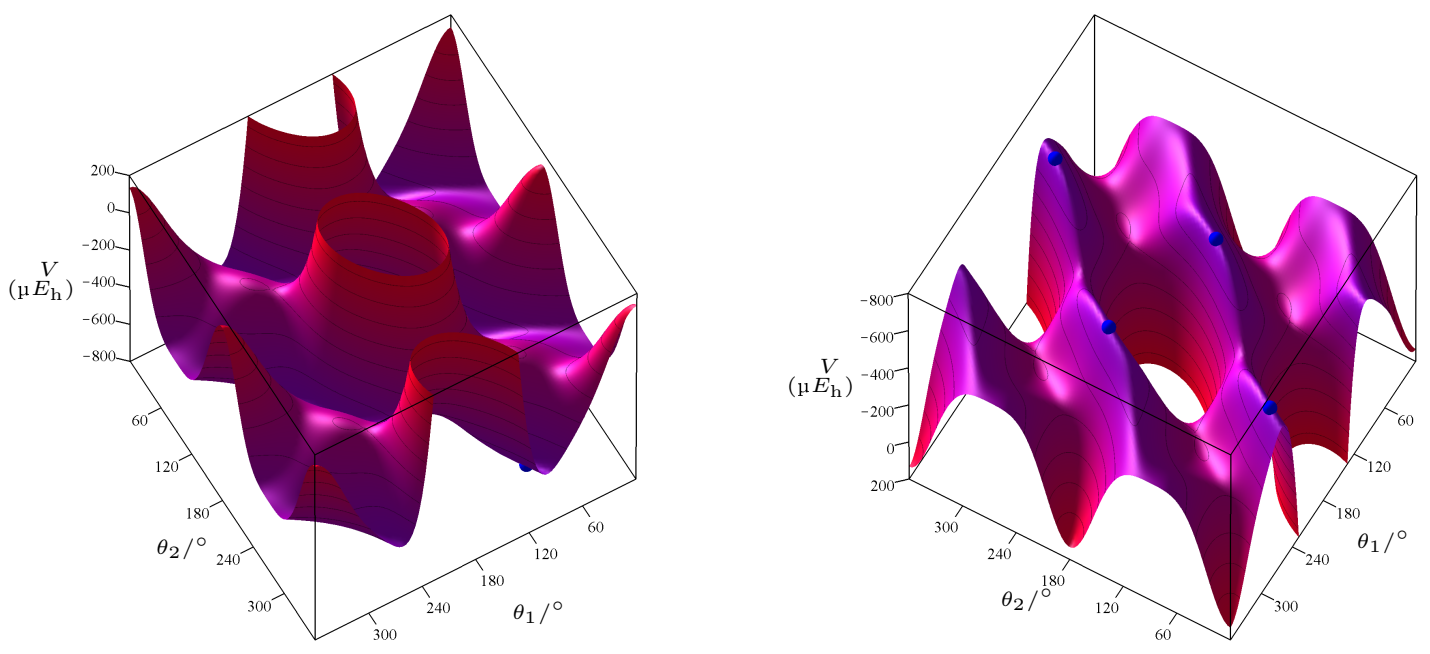
FIG. 4: A comparison of experimental (red) and calculated (black) intermolecular levels for COortho $\mathrm{N}_{2}$ in panel (a) and CO-para $\mathrm{N}_{2}$ in panel (b). Two sets of calculated levels are given: from this study and from Ref. 19 (italics). For each stack labelled by $K$, levels are given for $J=K$, $K+1, \cdots$ from the bottom up and the maximum $J$ is 2 . The zero of panel (b) for the calculated levels is at $2.7365 \mathrm{~cm}^{-1}$ relative to the scale of panel (a)

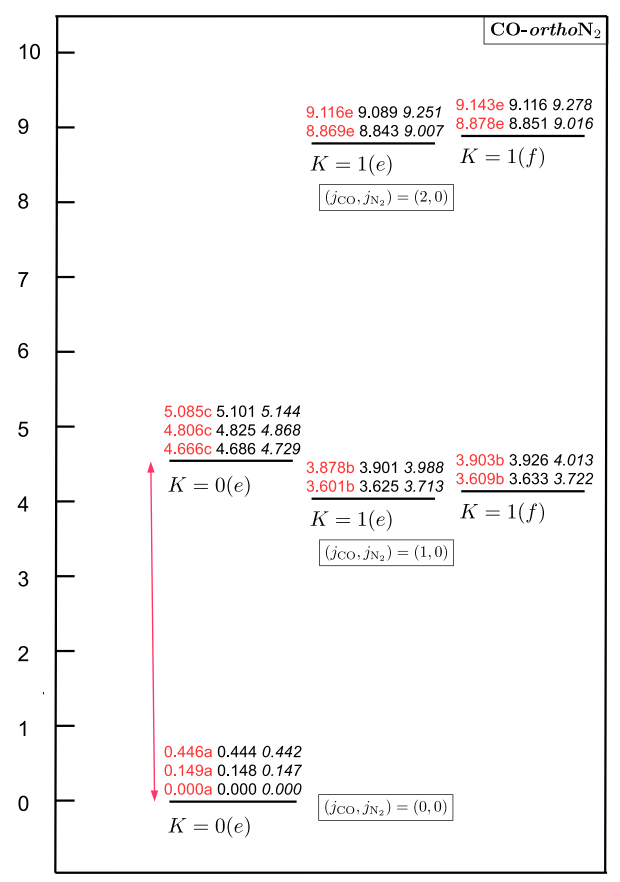

(a)

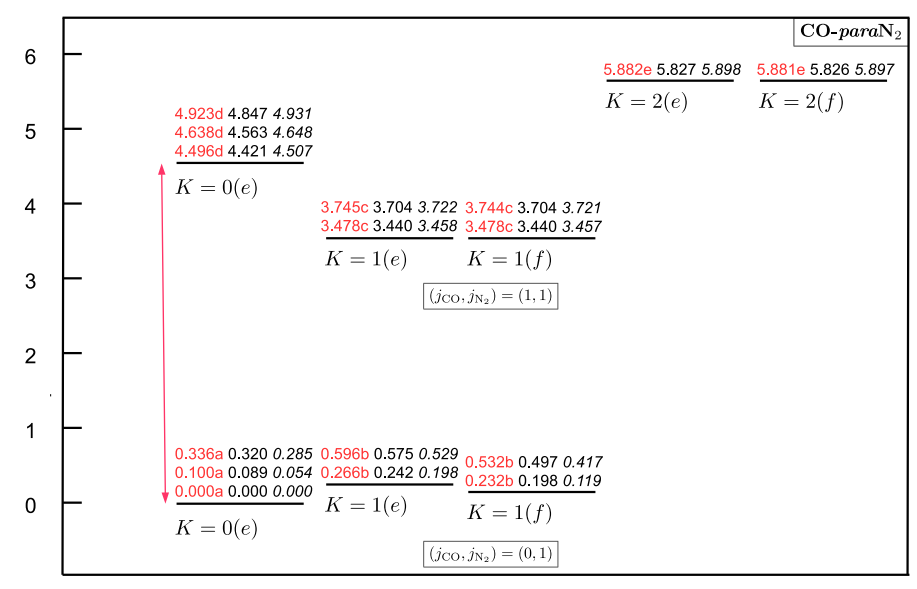

(b) 


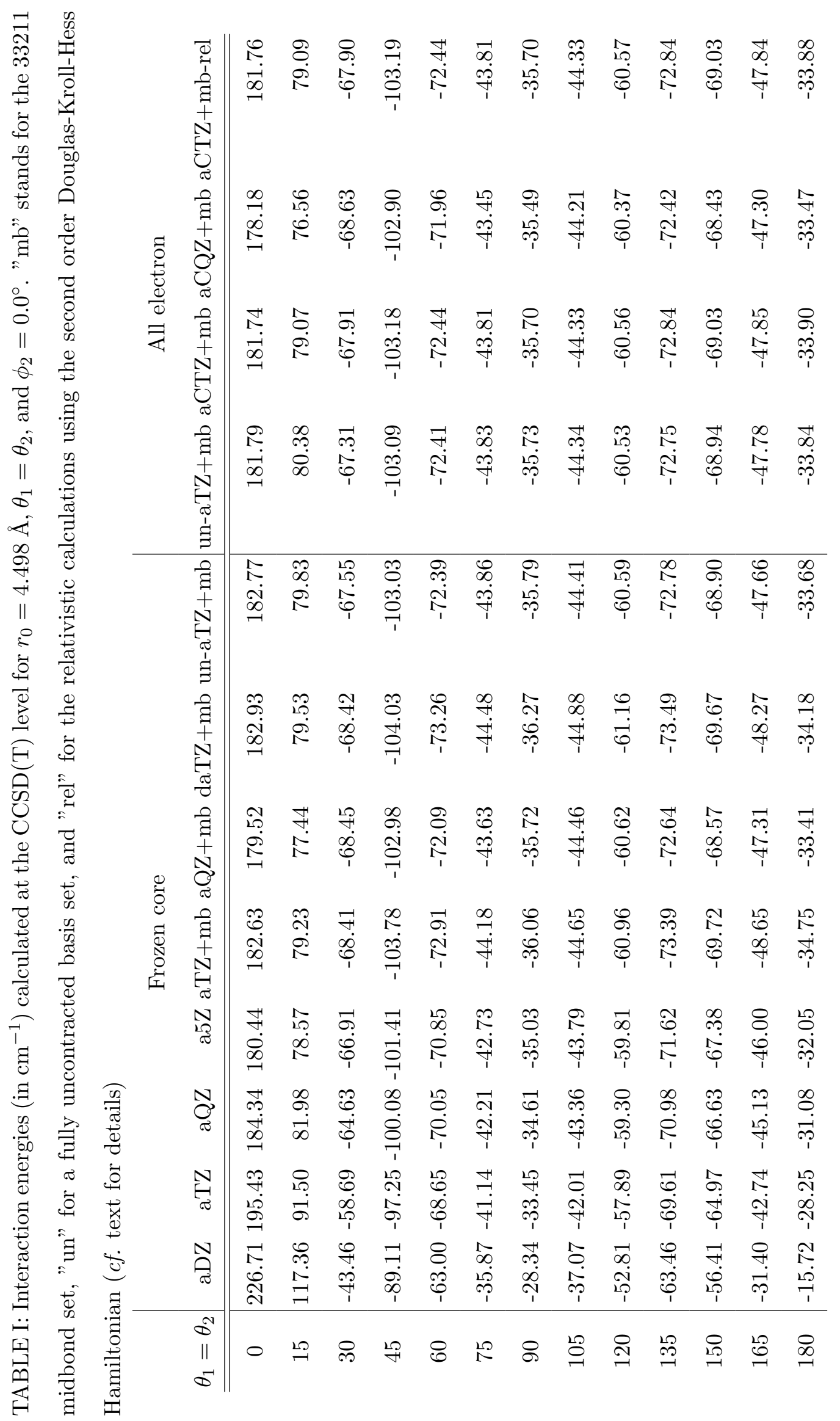


TABLE II: Parameters of the fit $V$. We write $e_{1} e_{2} e_{3} e_{4}$ as an abbreviation for $\left(e_{1}, e_{2}, e_{3}, e_{4}\right)$, e.g. $(0,0,1,0)$ simply becomes 0010

\begin{tabular}{rr|rr|rr}
\hline $\mathbf{e}$ & $c_{\mathrm{e}} / \mu E_{\mathrm{h}}$ & $\mathbf{e}$ & $c_{\mathrm{e}} / \mu E_{\mathrm{h}}$ & $\mathbf{e}$ & $c_{\mathrm{e}} / \mu E_{\mathrm{h}}$ \\
\hline 0010 & -32.12 & 0020 & 42713.23 & 0101 & -90922.33 \\
2000 & 45622.21 & 0011 & -43921.71 & 0110 & 94044.83 \\
1100 & -47238.94 & 0003 & -94238.97 & 1020 & 222526.29 \\
0201 & -151150.54 & 3000 & 23820.70 & 0012 & 99515.71 \\
1011 & -151182.26 & 0210 & 49958.19 & 0120 & -87836.88 \\
1110 & 113802.42 & 1200 & -27029.72 & 0040 & 134244.43 \\
0103 & -261135.27 & 2020 & 270883.97 & 0301 & -309531.19 \\
4000 & 161306.19 & 0031 & -143021.61 & 0112 & -57524.72 \\
2011 & 27878.69 & 3001 & 179528.32 & 0121 & 344483.45 \\
2002 & -226068.08 & 1201 & 553579.66 & 3100 & -534808.10 \\
1111 & -86760.14 & 2101 & -427291.73 & 2200 & 377662.86 \\
0050 & -38254.79 & 0302 & 128635.65 & 0401 & -68503.93 \\
5000 & -26919.29 & 0014 & -28092.38 & 1031 & 332771.36 \\
0212 & -243575.99 & 3011 & -56721.87 & 0023 & 69469.78 \\
1022 & -339359.60 & 2012 & 346112.63 & 0320 & -93145.80 \\
2003 & -33678.04 & 1130 & 114684.93 & 1202 & -583925.14 \\
3110 & 469398.51 & 1112 & -189641.53 & 2111 & 124011.68 \\
1310 & 86557.42 & 2102 & 486416.22 & 2201 & -480243.41 \\
3200 & 20583.83 & 0006 & 12990.26 & 0105 & -12025.49 \\
2040 & 6503.89 & 0501 & -27982.02 & 6000 & 27639.18 \\
0015 & -12989.92 & 2031 & -62247.86 & 0312 & 56617.25 \\
5001 & 6922.38 & 2022 & 92503.82 & 0321 & -76139.61 \\
4002 & -4820.57 & 1023 & -8329.71 & 0231 & -75468.23 \\
3003 & 30006.87 & 0141 & 20848.49 & 1140 & -7453.07 \\
1203 & 34335.28 & 4110 & 18736.81 & 1500 & -59868.76 \\
\hline 122 & 44846.43 & 1230 & -38456.68 & 2400 & 57833.68 \\
& & & & \\
\hline & & & & \\
\hline
\end{tabular}


TABLE III: Parameters for CO-N 2 . For TDM DVR parameters see Refs. 46 and 44

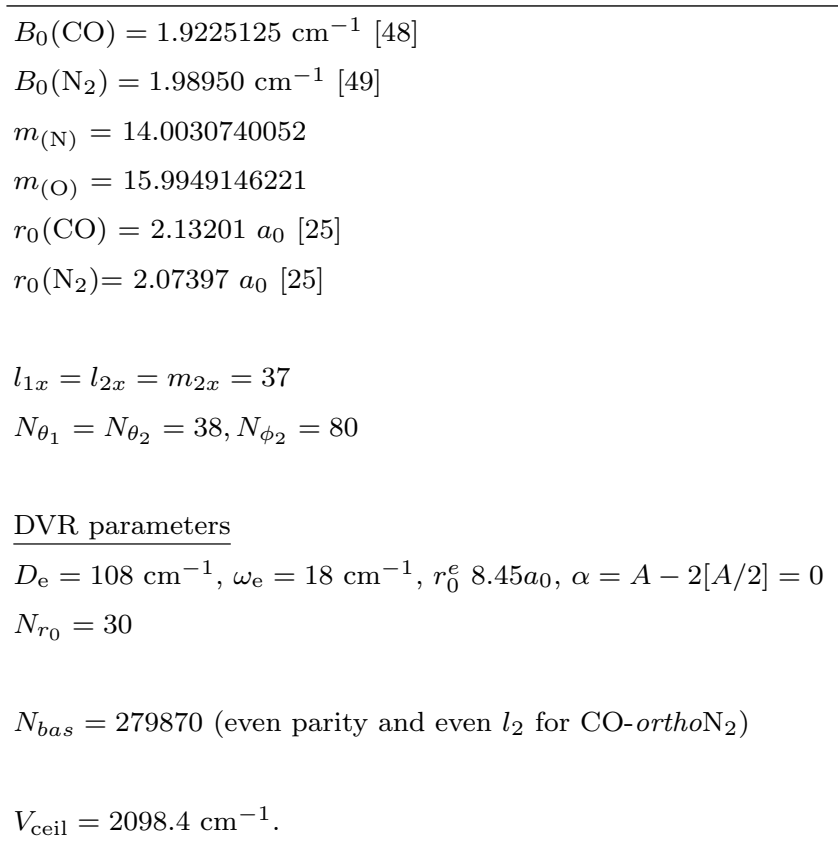


TABLE IV: Stack labels for the CO-ortho $\mathrm{N}_{2}$ and CO-para $\mathrm{N}_{2}$ observed $v_{\mathrm{CO}}=0$ states. The stacks are labelled in the order of increasing energy. The order of stacks for CO-ortho $\mathrm{N}_{2}$ follow that of the $v_{\mathrm{CO}}=1$ state [10] because more stacks are observed.

\begin{tabular}{llll}
\hline \hline CO- ortho $_{2}$ & label & $\left(j_{\mathrm{CO}}, j_{\mathrm{N}_{2}}, K, e / f\right)$ & Ref. \\
& a & $(0,0,0 e)$ & 8 \\
& b & $(1,0,1 e / f)$ & 8 \\
& c & $(1,0,0 e)$ & 8 \\
& d & $(2,0,2 e / f)$ & \\
& e & $(2,0,1 e / f)$ & 19 \\
& f & $(2,0,0 e / f)$ & \\
& g & $(1,2,0 e)$ & \\
& h & $(1,2,1 e / f)$ & \\
CO-para $\mathrm{N}_{2}$ & & & \\
& & $\left(j \mathrm{CO}, j_{\mathrm{N}_{2}}, K, e / f\right)$ & \\
& label & $(0,1,0 e)$ & 8 \\
& a & $(0,1,1 e / f)$ & 8 \\
& b & $(1,1,1 e / f)$ & 8 \\
c & $(1,1,0 e)$ & 10,19 \\
d & $(1,1,2 e / f)$ & 10,19 \\
\hline \hline
\end{tabular}


TABLE V: Comparison between the theoretical and the experimental $J \leq 10$ energy levels of CO-ortho $\mathrm{N}_{2}\left(\right.$ in $\left.\mathrm{cm}^{-1}\right)$. Stack labels are defined in Tab. IV. All levels are relative to the ZPE of CO- ortho $\mathrm{N}_{2}-76.1252 \mathrm{~cm}^{-1}$. PI group symmetry and spectroscopy parity $e / f$ are also indicated.

\begin{tabular}{|c|c|c|c|c|c|c|}
\hline \multicolumn{3}{|c|}{$J=0, A^{+}(e)$} & \multicolumn{4}{|c|}{$J=0, A^{-}(f)$} \\
\hline cal. & obs. & K stack & cal. & obs. I & & \\
\hline 0.0000 & 0.0000 & $0 \quad a$ & 16.0601 & & 0 & \\
\hline 4.6863 & 4.6663 & 0 & 23.7748 & & 0 & \\
\hline \multicolumn{3}{|c|}{$J=1, A^{+}(f)$} & \multicolumn{4}{|c|}{$J=1, A^{-}(e)$} \\
\hline cal. & obs. & K stack & cal. & obs. I & $\mathrm{K}$ & tack \\
\hline 3.6333 & 3.6093 & 1 & 0.1480 & 0.1486 & 0 & $a$ \\
\hline 8.8514 & 8.8780 & 1 & 3.6249 & 3.6009 & 1 & $b$ \\
\hline 13.5885 & & 1 & 4.8246 & 4.8057 & 0 & $c$ \\
\hline 15.4695 & & 1 & 8.8425 & 8.8691 & 1 & $e$ \\
\hline \multicolumn{3}{|c|}{$J=2, A^{+}(e)$} & \multicolumn{4}{|c|}{$J=2, A^{-}(f)$} \\
\hline cal. & obs. & K stack & cal. & obs. I & $\mathrm{K}$ & stack \\
\hline 0.4439 & 0.4457 & 0 & 3.9262 & 3.9034 & 1 & $b$ \\
\hline 3.9010 & 3.8781 & 1 & 9.1155 & 9.1425 & 1 & e \\
\hline 5.1013 & 5.0845 & 0 & 9.9916 & & 2 & \\
\hline 9.0893 & 9.1161 & 1 & 11.3735 & & 2 & \\
\hline \multicolumn{3}{|c|}{$J=3, A^{+}(f)$} & \multicolumn{4}{|c|}{$J=3, A^{-}(e)$} \\
\hline cal. & obs. & K stack & cal. & obs. & & stack \\
\hline 4.3652 & 4.3441 & 1 & 0.8875 & 0.8910 & 0 & $a$ \\
\hline 9.5128 & 9.5402 & 1 & 4.3146 & 4.2934 & 1 & $b$ \\
\hline 10.4358 & & 2 & 5.5162 & 5.5026 & 0 & $c$ \\
\hline 11.8065 & & 2 & 9.4616 & 9.4890 & 1 & $e$ \\
\hline \multicolumn{3}{|c|}{$J=4, A^{+}(e)$} & \multicolumn{4}{|c|}{$J=4, A^{-}(f)$} \\
\hline
\end{tabular}




\begin{tabular}{|c|c|c|c|c|}
\hline $1.4785 \quad 1.4843$ & $0 \quad a$ & $4.9497 \quad 4.9310$ & 1 & $b$ \\
\hline $4.8652 \quad 4.8464$ & $b$ & 10.043910 .0725 & 1 & $e$ \\
\hline $6.0693 \quad 6.0600$ & $c$ & 11.0262 & 2 & \\
\hline 9.96119 .9896 & $e$ & 12.3833 & 2 & \\
\hline \multicolumn{2}{|c|}{$J=5, A^{+}(f)$} & \multicolumn{3}{|c|}{$J=5, A^{-}(e)$} \\
\hline cal. obs. & $\mathrm{K}$ stack & cal. obs. & $\mathrm{K}$ st & tack \\
\hline $5.6792 \quad 5.6634$ & $b$ & $2.2163 \quad 2.2250$ & 0 & $a$ \\
\hline 10.709910 .7403 & $e$ & $5.5519 \quad 5.5363$ & 1 & $b$ \\
\hline 11.7615 & 2 & $6.7606 \quad 6.7565$ & 0 & $c$ \\
\hline 13.1033 & 2 & 10.589010 .6200 & 1 & $e$ \\
\hline \multicolumn{2}{|c|}{$J=6, A^{+}(e)$} & \multicolumn{3}{|c|}{$J=6, A^{-}(f)$} \\
\hline cal. obs. & K stack & cal. obs. & $\mathrm{K}$ st & tack \\
\hline $3.1003 \quad 3.1127$ & $a$ & $6.5527 \quad 6.5406$ & 1 & $b$ \\
\hline $6.3738 \quad 6.3623$ & $b$ & 11.511411 .5446 & 1 & $e$ \\
\hline $7.5900 \quad 7.5921$ & $c$ & 12.6406 & 2 & \\
\hline 11.346711 .3808 & $e$ & 13.9657 & 2 & \\
\hline \multicolumn{2}{|c|}{$J=7, A^{+}(f)$} & \multicolumn{3}{|c|}{$J=7, A^{-}(e)$} \\
\hline cal. obs. & K stack & cal. obs. & $\mathrm{K}$ st & tack \\
\hline $7.5694 \quad 7.5616$ & $b$ & $4.1300 \quad 4.1466$ & 0 & $a$ \\
\hline 12.448912 .4862 & $e$ & $7.3298 \quad 7.3234$ & 1 & $b$ \\
\hline 13.6627 & 2 & $8.5574 \quad 8.5664$ & 0 & $c$ \\
\hline 14.9699 & 2 & 12.234812 .2715 & 1 & $e$ \\
\hline \multicolumn{2}{|c|}{$J=8, A^{+}(e)$} & \multicolumn{3}{|c|}{$J=8, A^{-}(f)$} \\
\hline obs. & K stack & cal. obs. & $\mathrm{K}$ st & tack \\
\hline $5.3043 \quad 5.3257$ & $a$ & $8.7279 \quad 8.7250$ & 1 & $b$ \\
\hline $8.4187 \quad 8.4184$ & $b$ & 13.522713 .5652 & 1 & $e$ \\
\hline $9.6626 \quad 9.6795$ & $c$ & 14.8269 & 2 & \\
\hline 13.253713 .2885 & $e$ & 16.1147 & 2 & \\
\hline \multicolumn{2}{|c|}{$J=9, A^{+}(f)$} & \multicolumn{3}{|c|}{$J=9, A^{-}(e)$} \\
\hline
\end{tabular}




\begin{tabular}{|c|c|c|c|c|c|}
\hline 10.027010 .0294 & & $b$ & $6.6222 \quad 6.6488$ & . & $a$ \\
\hline 14.7331 & 1 & & $9.6391 \quad 9.6461$ & 1 & $b$ \\
\hline 16.1324 & 2 & & 10.905210 .9311 & 0 & $c$ \\
\hline$J=10, A^{+}$ & $(e)$ & & $J=10, A^{-}$ & $(f)$ & \\
\hline cal. obs. I & K & tack & cal. obs. & $\mathrm{K}$ & tack \\
\hline $8.0825 \quad 8.1140$ & 0 & $a$ & 11.465011 .4725 & 1 & $a$ \\
\hline 10.989711 .0050 & 1 & $b$ & 16.0800 & 1 & \\
\hline 12.284812 .3208 & 0 & $c$ & 17.5785 & 2 & \\
\hline
\end{tabular}


TABLE VI: Comparison between the theoretical and the experimental $J \leq 8$ energy levels of CO-para $\mathrm{N}_{2}\left(\right.$ in $\left.\mathrm{cm}^{-1}\right)$. Stack labels are defined in Tab. IV. The ZPE of CO-para $\mathrm{N}_{2}$ is $2.7365 \mathrm{~cm}^{-1}$ higher than that of $\mathrm{CO}-$ ortho $\mathrm{N}_{2}$. PI group symmetry and spectroscopy parity $e / f$ are also indicated.

\begin{tabular}{|c|c|c|c|c|c|c|c|}
\hline \multicolumn{4}{|c|}{$J=0, B^{+}(e)$} & \multicolumn{4}{|c|}{$J=0, B^{-}(f)$} \\
\hline cal. & obs. & $\mathrm{K}$ & tack & cal. & obs. & $\mathrm{K}$ & stack \\
\hline 0.0000 & 0.0000 & 0 & $a$ & 6.4110 & & 0 & \\
\hline 4.4205 & 4.4955 & 0 & $d$ & 15.1054 & & 0 & \\
\hline \multicolumn{4}{|c|}{$J=1, B^{+}(f)$} & \multicolumn{4}{|c|}{$J=1, B^{-}(e)$} \\
\hline cal. & obs. & $\mathrm{K}$ & tack & cal. & obs. & $\mathrm{K}$ & stack \\
\hline 0.1979 & 0.2324 & 1 & $b$ & 0.0887 & 0.1002 & 01 & $a$ \\
\hline 3.4397 & 3.4781 & 1 & $c$ & 0.2420 & 0.2655 & 10 & $b$ \\
\hline 6.5472 & & 0 & & 3.4398 & 3.4784 & 1 & $c$ \\
\hline 7.4532 & & 1 & & 4.5625 & 4.6382 & 0 & $d$ \\
\hline \multicolumn{4}{|c|}{$J=2, B^{+}(e)$} & \multicolumn{4}{|c|}{$J=2, B^{-}(f)$} \\
\hline cal. & obs. & $\mathrm{K}$ & tack & cal. & obs. & $\mathrm{K}$ & stack \\
\hline 0.3200 & 0.3360 & 01 & $a$ & 0.4970 & 0.5324 & 1 & $b$ \\
\hline 0.5751 & 0.5962 & 10 & $b$ & 3.7040 & 3.7445 & 1 & $c$ \\
\hline 3.7043 & 3.7453 & 1 & $c$ & 5.8264 & 5.8816 & 2 & $e$ \\
\hline 4.8465 & 4.9234 & 0 & $d$ & 6.8191 & & 0 & \\
\hline 5.8265 & 5.8818 & 2 & $e$ & 7.7598 & & 1 & \\
\hline \multicolumn{4}{|c|}{$J=3, B^{+}(f)$} & \multicolumn{4}{|c|}{$J=3, B^{-}(e)$} \\
\hline cal. & obs. & $\mathrm{K}$ & tack & cal. & obs. & $\mathrm{K}$ & stack \\
\hline 0.9453 & 0.9822 & 1 & $b$ & 0.6932 & 0.7128 & 0 & $a$ \\
\hline 4.1011 & 4.1445 & 1 & $c$ & 1.0476 & 1.0684 & 1 & $b$ \\
\hline 6.2848 & 6.3405 & 2 & $e$ & 4.1016 & 4.1461 & 1 & $c$ \\
\hline 7.2258 & & 0 & & 5.2724 & 5.3510 & 0 & $d$ \\
\hline 8.2201 & & 1 & & 6.2854 & 6.3411 & 2 & $e$ \\
\hline
\end{tabular}




\begin{tabular}{|c|c|c|c|c|c|c|}
\hline \multicolumn{3}{|c|}{$J=4, B^{+}(e)$} & \multicolumn{4}{|c|}{$J=4, B^{-}(f)$} \\
\hline cal. & obs. & $\mathrm{K}$ stack & & obs. & & stack \\
\hline 1.2080 & 1.2313 & 0 & 1.5426 & 1.5813 & 1 & $b$ \\
\hline 1.6588 & 1.6803 & 1 & 4.6314 & 4.6789 & 1 & $c$ \\
\hline 4.6320 & 4.6812 & 1 & 6.8943 & 6.9509 & 2 & $e$ \\
\hline 5.8397 & 5.9208 & 0 & 7.7662 & & 0 & \\
\hline 6.8961 & 6.9526 & 2 & 8.8340 & & 1 & \\
\hline \multicolumn{3}{|c|}{$J=5, B^{+}(f)$} & \multicolumn{4}{|c|}{$J=5, B^{-}(e)$} \\
\hline cal. & obs. & K stack & cal. & obs. & $\mathrm{K}$ & stack \\
\hline 2.2883 & 2.3295 & 1 & 1.8644 & 1.8916 & 0 & $a$ \\
\hline 5.2955 & 5.3481 & 1 & 2.4074 & 2.4305 & 1 & $b$ \\
\hline 7.6535 & 7.7114 & 2 & 5.2962 & 5.3511 & 1 & $c$ \\
\hline 8.4395 & & 0 & 6.5482 & 6.6324 & 0 & $d$ \\
\hline 9.6013 & & 1 & 7.6578 & 7.7155 & 2 & $e$ \\
\hline \multicolumn{3}{|c|}{$J=6, B^{+}(e)$} & \multicolumn{4}{|c|}{$J=6, B^{-}(f)$} \\
\hline cal. & obs. & $\mathrm{K}$ stack & cal. & obs. & $\mathrm{K}$ & stack \\
\hline 2.6618 & 2.6935 & 0 & 3.1818 & 3.2259 & 1 & $b$ \\
\hline 3.2924 & 3.3177 & 1 & 6.0942 & 6.1527 & 1 & $c$ \\
\hline 6.0945 & 6.1562 & 1 & 8.5608 & 8.6204 & 2 & $e$ \\
\hline 7.3975 & 7.4855 & 0 & 9.2448 & & 0 & \\
\hline 8.5695 & 8.6288 & 2 & 10.5216 & & 1 & \\
\hline \multicolumn{3}{|c|}{$J=7, B^{+}(f)$} & \multicolumn{4}{|c|}{$J=7, B^{-}(e)$} \\
\hline cal. & obs. & $\mathrm{K}$ stack & cal. & obs. & $\mathrm{K}$ & tack \\
\hline 4.2224 & 4.2701 & 1 & 3.5997 & 3.6365 & 0 & $a$ \\
\hline 7.0277 & 7.0933 & 1 & 4.3121 & 4.3406 & 1 & $b$ \\
\hline 9.6134 & 9.6758 & 2 & 7.0274 & 7.0969 & 1 & $c$ \\
\hline 10.1823 & & 0 & 8.3872 & 8.4797 & 0 & $d$ \\
\hline 11.5940 & & 1 & 9.6302 & 9.6915 & 2 & $e$ \\
\hline \multicolumn{3}{|c|}{$J=8, B^{+}(e)$} & \multicolumn{4}{|c|}{$J=8, B^{-}(f)$} \\
\hline
\end{tabular}




\begin{tabular}{llllllll}
4.6774 & 4.7199 & 0 & $a$ & 5.4090 & 5.4609 & 1 & $b$ \\
5.4652 & 5.4976 & 1 & $b$ & 8.0966 & 8.1703 & 1 & $c$ \\
8.0951 & 8.1734 & 1 & $c$ & 10.8068 & 10.8743 & 2 & $e$ \\
9.5167 & 9.6145 & 0 & $d$ & 11.2540 & 1 & \\
10.8387 & 10.9021 & 2 & $e$ & 12.8170 & 1 & \\
\hline \hline
\end{tabular}


The table of contents entry: We present a highly accurate $a b$ initio intermolecular potential-energy surface and rovibrational spectrum for the $\mathrm{CO}-\mathrm{N}_{2}$ complex.

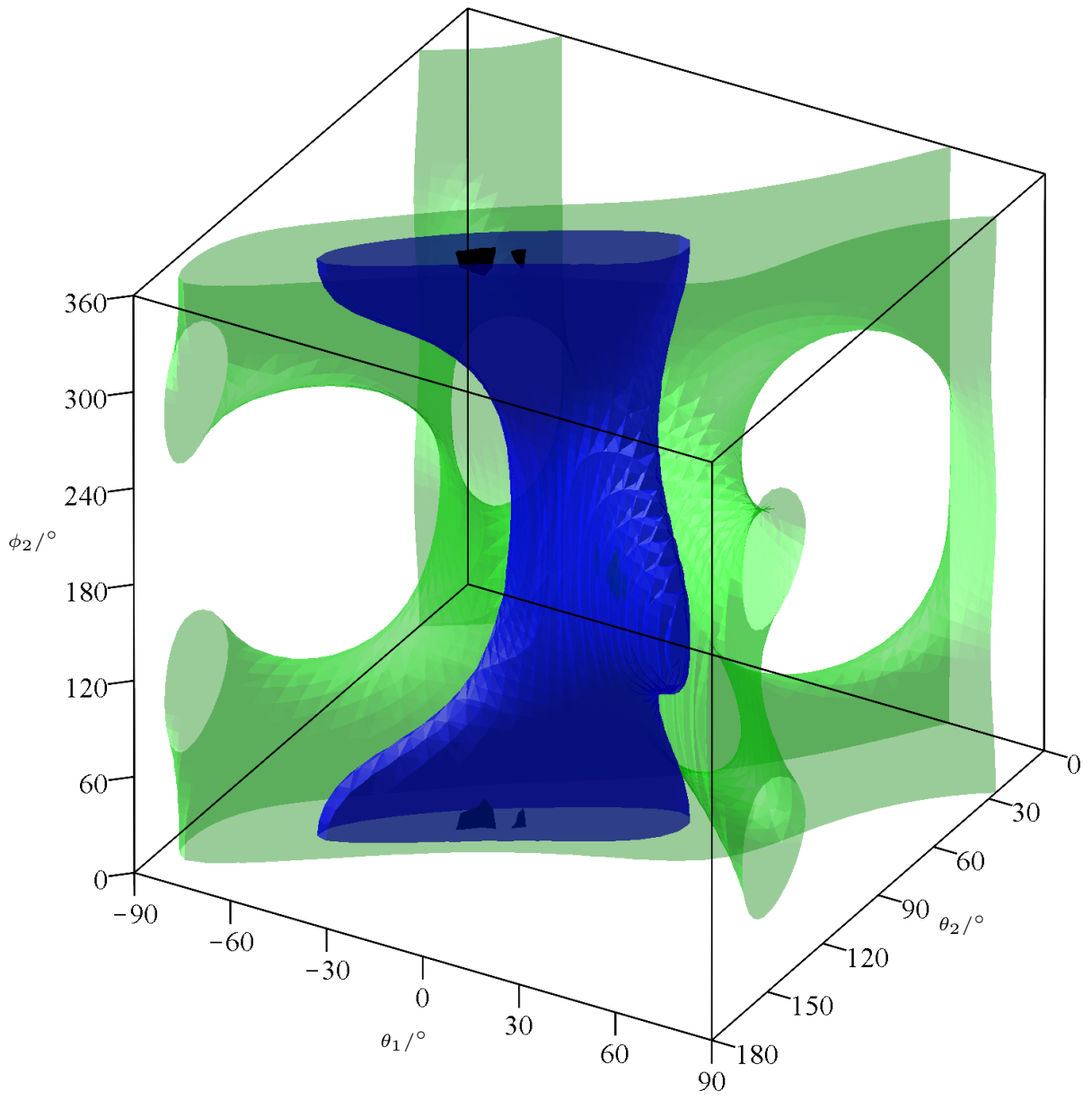

